\title{
LeARning, AdAPTIVe Expectations, ANd TechnOLOGY SHOCKS
}

$$
\text { by }
$$

Kevin X.D. Huang, Zheng Liu, and Tao Zha

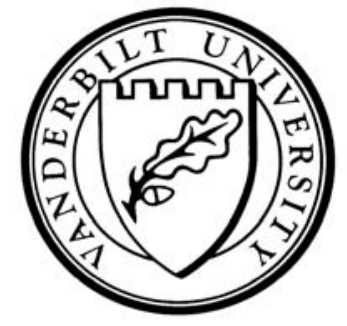

Working Paper No. 08-W07

April 2008

\section{DEPARTMENT OF ECONOMICS \\ VANDERBILT UNIVERSITY \\ NASHVILLE, TN 37235}

www.vanderbilt.edu/econ 


\title{
LEARNING, ADAPTIVE EXPECTATIONS, AND TECHNOLOGY SHOCKS
}

\author{
KEVIN X.D. HUANG, ZHENG LIU, AND TAO ZHA
}

\begin{abstract}
This study explores theoretical and macroeconomic implications of the self-confirming equilibrium in a standard growth model. When rational expectations are replaced by adaptive expectations, we prove that the self-confirming equilibrium is the same as the steady state rational expectations equilibrium, but that dynamics around the steady state are substantially different between the two equilibria. We show that, in contrast to Williams (2003), the differences are driven mainly by the lack of the wealth effect and the strengthening of the intertemporal substitution effect, not by escapes. As a result, adaptive expectations substantially alter the amplification and propagation mechanisms and allow technology shocks to exert much more impact on macroeconomic variables than do rational expectations.
\end{abstract}

\section{INTRODUCTION}

Adaptive learning models in macroeconomics have been used for many applications (Sargent, 2007). ${ }^{1}$ We focus, in this paper, on yet another application in the context of a standard growth model in which rational expectations are replaced by adaptive expectations. The stability of rational expectations under learning in real business cycle (RBC) models has been studied in the literature (Evans and Honkapohja, 2001;

Date: March 30, 2008.

Key words and phrases. Self confirming equilibrium; amplification; labor market dynamics; wealth and substitution effects; hump-shaped responses.

This paper is prepared for the 2008 Annual Conference of the Royal Economic Society. We thank Marty Eichenbaum, Martin Ellison, Selo Imrohoroglu, Tom Sargent, and Paolo Surico for helpful comments. The views expressed herein are those of the authors and do not necessarily reflect the views of the Federal Reserve Bank of Atlanta or the Federal Reserve System.

${ }^{1}$ To give a few examples, Lucas (1986), Marcet and Sargent (1989), and Evans and Honkapohja (2001) recommend selecting rational expectations equilibria that are stable under least squares learning; Primiceri (2006), Sargent, Williams, and Zha (2006b), and Carboni and Ellison (2008) use learning mechanisms to explain the rise and fall of American inflation; Adam, Marcet, and Nicolini (2008) show how learning helps improve the fit of the model of asset pricing. 
Bullard and Duffy, 2004). In a closely related paper, Williams (2003) considers a variety of standard learning rules in a RBC model and finds that learning dynamics differ very little from rational expectations dynamics. As noted by Williams (2003), those learning rules do not separate agents' beliefs and their decision making. The only role for agents is forecasting by using a reduced-form model. Consequently, one would conclude that learning dynamics do not teach us anything new, as compared to the rational expectations version of the RBC model.

In this paper, we reexamine this conclusion in the standard growth model with both neutral and investment specific technologies. Following the commonly-used learning mechanism studied by Marcet and Nicolini (2003) and Sargent, Williams, and Zha (2006a), we examine the implications of misspecified learning by separating agents' beliefs and their decision rules. ${ }^{2}$ Rational expectations are simply replaced by adaptive expectations, while all decision equations under rational expectations remain intact. We show that this slight departure from rational expectations has important ramifications by answering the following questions:

- Does there exist a self-confirming equilibrium (SCE) in our learning environment? Is it unique?

- Are there strong escape dynamics away from the domain of attraction of the SCE?

- How does learning amplify the effects of technology shocks, in contrast to rational expectations?

- How does learning affect the relative importance of investment-specific to neutral technology shocks?

To answer these questions, we obtain the closed-form solutions for both rational expectations and learning models. These analytical solutions enable us to prove the existence and uniqueness of the SCE under all admissible parameterizations in our learning model. We further prove that the SCE coincides with the steady state

\footnotetext{
${ }^{2}$ Williams (2003) studies a different kind of misspecified learning in which agents do not know the true parameters of the production function. By assuming full depreciation of the capital stock, an i.i.d. technology process, and inelastic labor, he shows that learning leads to occasional, but recurrent, large deviations away from an SCE, called "escape dynamics." For other studies of escape dynamics, see Sargent (1999), Cho, Williams, and Sargent (2002), Kasa (2004), and Adam, Evans, and Honkapohja (2006).
} 
rational expectations equilibrium (REE), but that learning dynamics are substantially different from rational expectations dynamics. Unlike Marcet and Nicolini (2003), Williams (2003), and Sargent, Williams, and Zha (2006a), however, we show that learning dynamics are stationary and that the differences between learning dynamics and rational-expectations dynamics are not driven by escape dynamics.

These theoretical results enable one to draw macroeconomic implications from our learning model. The dynamic responses of output, consumption, investment, and labor hours, following a neutral technology shock, are substantially larger in the adaptive expectations model than in the rational expectations model. Because agents under adaptive expectations form expectations of future income based on the past observations, introducing learning turns off the channel of the wealth effect and reinforces the intertemporal substitution effect. Consequently, it helps amplify the effects of the neutral shock on many aggregate variables and improve the model's predictions on the labor market dynamics. In the rational expectations model, equilibrium hours change too little and the equilibrium real wage fluctuates too much. In contrast, learning amplifies the response of labor and at the same time dampens the response of the real wage.

As for responses to a positive biased technology shock, the muted wealth effect under adaptive learning, in combination with the strong intertemporal substitution effect, helps amplify the responses of all macroeconomic variables, including the real wage, as compared to the rational expectations model. In terms of contributions to fluctuations in output, consumption, and investment, the relative importance of the biased technology shock to the neutral technology shock under learning is much larger than that under rational expectations.

In contrast to the rational expectations model, the dynamic responses of hours to both technology shocks can be negative after initial periods. The less persistent these shocks are, the more pronounced such negative responses of hours can become. The negative responses are a result of hump-shaped responses. The learning model is more likely to generate hump-shaped responses of consumption, investment, real wage, and hours, the less persistent technology shocks are. 
Overall, our results indicate that the growth model with adaptive expectations is capable of giving technology shocks a much more important role in causing fluctuations of key macroeconomic variables than is the rational expectations model.

\section{THE MODEL}

In this section, we describe the standard growth model with both neutral and biased technologies. The economy is populated by a continuum of infinitely lived and identical households. The representative household is endowed with a unit of time. The household derives utility from consumption and leisure, with the utility function

$$
\mathrm{E}_{0} \sum_{t=0}^{\infty} \beta^{t}\left\{\ln C_{t}-\xi \frac{L_{t}^{1+\eta}}{1+\eta}\right\},
$$

where $C_{t}$ denotes consumption, $L_{t}$ denotes labor hours, $\beta \in(0,1)$ denotes the subjective discount factor, and $\mathrm{E}_{0}$ denotes an expectation at the initial time 0 .

The economy is also populated by a continuum of identical, perfectly competitive firms. The representative firm has access to a constant returns to scale technology represented by the production function

$$
Y_{t}=K_{t-1}^{1-\alpha}\left(Z_{t} L_{t}\right)^{\alpha}
$$

where $Y_{t}$ denotes output, $K_{t-1}$ denotes capital input, and $L_{t}$ denotes labor input. The term $Z_{t}$ denotes the neutral technological change and follows the stochastic process

$$
Z_{t}=\lambda_{z}^{t} \nu_{t}
$$

where $\lambda_{z}$ is the trend component and $\nu_{t}$ is the stationary component that follows the $\mathrm{AR}(1)$ process

$$
\ln \nu_{t}=\rho_{\nu} \ln \nu_{t-1}+\varepsilon_{\nu t} .
$$

The persistence parameter $\rho_{\nu} \in(0,1]$ and the shock $\varepsilon_{\nu t}$ is a white noise process with mean zero and variance $\sigma_{\nu}^{2}$. The shock process specified in (3)-(4) implies that, if $0<\rho_{\nu}<1$, then the neutral technology follows a stationary stochastic process with a deterministic trend; if $\rho_{\nu}=1$, then the neutral technology follows a random walk process with a drift. ${ }^{3}$

\footnotetext{
${ }^{3}$ In the case with $\rho_{\nu}=1$, we have $Z_{t}=\lambda_{z}^{t} \nu_{t}$ and $\nu_{t}=\nu_{t-1} \exp \left(\varepsilon_{\nu t}\right)$, or equivalently, $Z_{t}=$ $Z_{t-1} \lambda_{z} \exp \left(\varepsilon_{\nu t}\right)$.
} 
The economy has an initial stock of capital denoted by $K_{-1}$. Capital stock evolves over time according to the law of motion

$$
K_{t}=(1-\delta) K_{t-1}+Q_{t} I_{t}
$$

where $K_{t}$ denotes the period- $t$ capital stock, $I_{t}$ denotes investment, $Q_{t}$ denotes the relative price of investment goods, and the parameter $\delta \in(0,1)$ denotes the capital depreciation rate. Following Greenwood, Hercowitz, and Krusell (1997), we assume that $Q_{t}$ represents the (inverse of) investment specific technological change. Similar to the neutral technology, we assume that the investment-specific technology shock $Q_{t}$ follows the stochastic process

$$
Q_{t}=\lambda_{q}^{t} \mu_{t}
$$

where $\lambda_{q}$ is the trend component and $\mu_{t}$ is the stationary component that follows the $\operatorname{AR}(1)$ process

$$
\ln \mu_{t}=\rho_{\mu} \ln \mu_{t-1}+\varepsilon_{\mu t}
$$

The persistence parameter $\rho_{\mu} \in(0,1)$ and the innovation term $\varepsilon_{\mu t}$ is white-noise process with mean zero and variance $\sigma_{\mu}^{2}$. Again, our specification of the $Q_{t}$ process here nests the random-walk process as a special case with $\rho_{\mu}=1$.

The aggregate resource constraint is given by

$$
C_{t}+I_{t}=Y_{t}
$$

\section{Equilibrium Allocation and Balanced Growth}

Since the model economy has perfect competition and no externality, the First Welfare Theorem applies. Thus, the equilibrium allocations are Pareto efficient and can be found by solving a social planner's problem.

The social planner maximizes the representative household's utility (1) subject to the resource constraint (8) and the capital law of motion (5). The first order conditions imply that

$$
\begin{aligned}
\xi L_{t}^{1+\eta} & =\alpha Y_{t} / C_{t} \\
1 & =\beta \mathrm{E}_{t}\left\{\frac{Q_{t}}{Q_{t+1}} \frac{C_{t}}{C_{t+1}}\left[1-\delta+Q_{t+1}(1-\alpha) \frac{Y_{t+1}}{K_{t}}\right]\right\} .
\end{aligned}
$$


On the balanced growth path, $C_{t}, I_{t}$, and $Y_{t}$ grow at the same rate of $\lambda_{z} \lambda_{q}^{(1-\alpha) / \alpha}$ while the capital stock $K_{t}$ grows at a faster rate of $\lambda_{z} \lambda_{q}^{1 / \alpha}$. We define the following stationary variables ${ }^{4}$

$$
\tilde{Y}_{t}=\frac{Y_{t}}{Z_{t} Q_{t}^{(1-\alpha) / \alpha}}, \tilde{C}_{t}=\frac{C_{t}}{Z_{t} Q_{t}^{(1-\alpha) / \alpha}}, \tilde{I}_{t}=\frac{I_{t}}{Z_{t} Q_{t}^{(1-\alpha) / \alpha}}, \quad \tilde{K}_{t}=\frac{K_{t}}{Z_{t} Q_{t}^{1 / \alpha}}
$$

Given these stationary variables, we can rewrite the equilibrium conditions (2), (5), (8), (9), and (10) as

$$
\begin{gathered}
\tilde{Y}_{t}\left(\frac{Z_{t}}{Z_{t-1}}\right)^{1-\alpha}\left(\frac{Q_{t}}{Q_{t-1}}\right)^{(1-\alpha) / \alpha}=\tilde{K}_{t-1}^{1-\alpha} L_{t}^{\alpha} \\
\tilde{K}_{t} \frac{Z_{t}}{Z_{t-1}}\left(\frac{Q_{t}}{Q_{t-1}}\right)^{1 / \alpha}=(1-\delta) \tilde{K}_{t-1}+\tilde{I}_{t} \frac{Z_{t}}{Z_{t-1}}\left(\frac{Q_{t}}{Q_{t-1}}\right)^{1 / \alpha}, \\
\tilde{C}_{t}+\tilde{I}_{t}=\tilde{Y}_{t} \\
\xi L_{t}^{1+\eta}=\alpha \tilde{Y}_{t} / \tilde{C}_{t} \\
1=\beta \mathrm{E}_{t}\left[(1-\delta) \frac{\tilde{C}_{t}}{\tilde{C}_{t+1}} \frac{Z_{t}}{Z_{t+1}}\left(\frac{Q_{t}}{Q_{t+1}}\right)^{1 / \alpha}+(1-\alpha) \frac{\tilde{C}_{t}}{\tilde{C}_{t+1}} \frac{\tilde{Y}_{t+1}}{\tilde{K}_{t}}\right] .
\end{gathered}
$$

Denote $\lambda_{k} \equiv \lambda_{z} \lambda_{q}^{1 / \alpha}$. It follows from the above conditions that the steady state equilibrium can be described by the following equations

$$
\begin{gathered}
\lambda_{k}^{1-\alpha} \tilde{Y}=\tilde{K}^{1-\alpha} L^{\alpha}, \\
i_{k}=\frac{\tilde{I}}{\tilde{K}}=1-\frac{1-\delta}{\lambda_{k}}, \\
\tilde{C}+\tilde{I}=\tilde{Y} \\
\xi L^{1+\eta}=\alpha \tilde{Y} / \tilde{C} \\
y_{k}=\frac{\tilde{Y}}{\tilde{K}}=\frac{1}{\beta(1-\alpha)}\left[1-\frac{\beta(1-\delta)}{\lambda_{k}}\right] .
\end{gathered}
$$

\footnotetext{
${ }^{4} \mathrm{An}$ alternative approach to induce stationarity in the model is to detrend the variable by its deterministic trend. For instance, one can define $\tilde{X}_{t}=\frac{X_{t}}{\lambda_{x}^{t}}$, where $X_{t} \in\left\{Y_{t}, C_{t}, I_{t}, K_{t}\right\}$ and $\lambda_{x}$ is a function of $\lambda_{z}$ and $\lambda_{q}$. Our approach has an advantage in that it nests the model with stochastic trends (e.g., random walk processes) as a special case while the other approach does not.
} 
The consumption-output and investment-output ratios can derived from the above steady state conditions:

$$
\begin{gathered}
i_{y}=\frac{\tilde{I}}{\tilde{Y}}=\beta(1-\alpha) \frac{\lambda_{k}-(1-\delta)}{\lambda_{k}-\beta(1-\delta)}, \\
c_{y}=\frac{\tilde{C}}{\tilde{Y}}=1-i_{y} .
\end{gathered}
$$

Log-linearizing the equilibrium conditions (11), (12), (13), (14), and (15) and rearranging the terms, we obtain the following five equations describing the production function, the law of motion for capital accumulation, the resource constraint, the optimal consumption-labor-supply decision, and the optimal investment decision:

$$
\begin{gathered}
\hat{y}_{t}-\alpha \hat{l}_{t}+(1-\alpha)\left(\frac{1}{\alpha} \Delta \hat{\mu}_{t}+\Delta \hat{\nu}_{t}\right)=(1-\alpha) \hat{k}_{t-1}, \\
\hat{k}_{t}-i_{k} \hat{i}_{t}+\left(1-i_{k}\right)\left(\alpha^{-1} \Delta \hat{\mu}_{t}+\Delta \hat{\nu}_{t}\right)=\left(1-i_{k}\right) \hat{k}_{t-1}, \\
c_{y} \hat{c}_{t}+i_{y} \hat{i}_{t}=\hat{y}_{t} \\
\hat{y}_{t}=\hat{c}_{t}+(1+\eta) \hat{l}_{t} \\
\beta(1-\alpha) y_{k} \hat{k}_{t}-\hat{c}_{t}+\left[1-\beta(1-\alpha) y_{k}\right]\left(\frac{\rho_{\mu}-1}{\alpha} \hat{\mu}_{t}+\left(\rho_{\nu}-1\right) \hat{\nu}_{t}\right)= \\
{\left[\beta(1-\alpha) c_{k}-1\right] E_{t} \hat{c}_{t+1}+\beta(1-\alpha) i_{k} E_{t} \hat{i}_{t+1},}
\end{gathered}
$$

where $\Delta$ is the first difference operator (e.g., $\Delta z_{t}=z_{t}-z_{t-1}$ ), the notation $\hat{x}_{t}$ denotes $\ln \tilde{X}_{t}-\ln \tilde{X}$ for $X=C, I, Y, K$ or $\ln X_{t}-\ln X$ for $X=L, i_{k}, c_{y}, i_{y}$, and $y_{k}$ are steady-state ratios defined in (17), (22), (21), (20), and $c_{k}=\frac{\tilde{C}}{\tilde{K}}$ is derived as

$$
\beta(1-\alpha)\left(c_{k}+1\right)=1-\frac{\alpha \beta(1-\delta)}{\lambda_{k}} .
$$

Definition 1. Admissible values of the deep parameters are $\beta \in(0,1), \eta \geq 0, \alpha \in$ $(0,1), \delta \in[0,1], \lambda_{z} \geq 1$, and $\lambda_{q} \geq 1$.

In the literature, dynamics are often simulated for a particular set of admissible values of the deep parameters by numerically solving the rational-expectations equilibrium system given by the above conditions. We shall show, however, that the equilibrium characterized by (23)-(27) can be solved analytically for all admissible values of the deep parameters. The crucial step is to derive a stochastic process for capital, as stated in the following proposition. 
Proposition 1. The equilibrium solution for capital satisfies the following second-order stochastic difference equation:

$$
\hat{k}_{t}=\gamma_{1} E_{t} \hat{k}_{t+1}+\gamma_{2} \hat{k}_{t-1}+\gamma_{\mu 1} \hat{\mu}_{t}+\gamma_{\nu 1} \hat{\nu}_{t}+\gamma_{\mu 2} \hat{\mu}_{t-1}+\gamma_{\nu 2} \hat{\nu}_{t-1}
$$

where the coefficients $\gamma_{1}, \gamma_{2}, \gamma_{\mu 1}, \gamma_{\nu 1}, \gamma_{\mu 2}$, and $\gamma_{\nu 2}$ are reported in Appendix A.

Proof. See Appendix B.

Proposition 1 is the key to obtaining all of our theoretical results, as is shown in the next section.

\section{REE vs. SCE: Analytical Results}

In this section, we derive the closed-form solutions for both the REE and the SCE. The key is to solve (29); the solution depends on how agents form expectations of the endogenous accumulation process of capital. Once this solution is obtained, it is relatively straightforward to derive the closed-form solutions for the other variables, which are reported in Appendix A.

For the REE solution, we have the following result.

Proposition 2. The solution to the second-order differential equation (29) under the rational expectations assumption is

$$
\hat{k}_{t}=a \hat{k}_{t-1}+b \hat{\nu}_{t}+c \hat{\mu}_{t}+d \hat{\nu}_{t-1}+e \hat{\mu}_{t-1}
$$

where

$$
\begin{gathered}
a=\frac{1-\sqrt{1-4 \gamma_{1} \gamma_{2}}}{2 \gamma_{1}}, \quad b=\frac{\gamma_{1} d+\gamma_{\nu 1}}{1-\left(\rho_{\nu}+a\right) \gamma_{1}}, \quad c=\frac{\gamma_{1} e+\gamma_{\mu 1}}{1-\left(\rho_{\mu}+a\right) \gamma_{1}}, \\
d=\frac{\gamma_{\nu 2}}{1-\gamma_{1} a}, \quad e=\frac{\gamma_{\mu 2}}{1-\gamma_{1} a} .
\end{gathered}
$$

Furthermore, this solution is stationary and unique.

Proof. See Appendix C.

Given the shock processes and an initial condition for capital, (30) gives the dynamic solution for capital. For a comparison with the SCE solution, this dynamic solution can be expressed as 


$$
\hat{k}_{t}=b \varepsilon_{\nu, t}+c \varepsilon_{\mu, t}+\sum_{i=1}^{\infty}\left(b b_{i}+d b_{i-1}\right) \varepsilon_{\nu, t-i}+\sum_{i=1}^{\infty}\left(c c_{i}+e c_{i-1}\right) \varepsilon_{\mu, t-i},
$$

where for all $i \geq 0$,

$$
\begin{aligned}
& b_{i}=\sum_{j=0}^{i} a^{i-j} \rho_{\nu}^{j}, \quad \text { while if } \quad \rho_{\nu} \neq a \quad \text { then } \quad b_{i}=\frac{a^{i+1}-\rho_{\nu}^{i+1}}{a-\rho_{\nu}} ; \\
& c_{i}=\sum_{j=0}^{i} a^{i-j} \rho_{\mu}^{j}, \quad \text { while if } \quad \rho_{\mu} \neq a \quad \text { then } \quad c_{i}=\frac{a^{i+1}-\rho_{\mu}^{i+1}}{a-\rho_{\mu}} ;
\end{aligned}
$$

We now assume that agents have adaptive expectations. We follow Marcet and Nicolini (2003) and Sargent, Williams, and Zha (2006a) to replace $E_{t} \hat{k}_{t+1}$ by $\hat{E}_{t} \hat{k}_{t+1}$ such that

$$
\hat{E}_{t} \hat{k}_{t+1}=\hat{\beta}_{t}
$$

Agents update their beliefs $\hat{\beta}_{t}$ using the following constant-gain learning (CGL) algorithm:

$$
\hat{\beta}_{t}=\hat{\beta}_{t-1}+g\left(\hat{k}_{t-1}-\hat{\beta}_{t-1}\right)
$$

where $0<g<<1$ is a gain representing how fast past observations are discounted in the learning regression.

The dynamics of $\hat{k}_{t}$ produced by (29) under the above learning algorithm (32) follow the process

$$
\hat{k}_{t}=\gamma_{1} \hat{\beta}_{t}+\gamma_{2} \hat{k}_{t-1}+\gamma_{\nu 1} \hat{\nu}_{t}+\gamma_{\mu 1} \hat{\mu}_{t}+\gamma_{\nu 2} \hat{\nu}_{t-1}+\gamma_{\mu 2} \hat{\mu}_{t-1}
$$

In self-confirming equilibrium, beliefs are not contradicted by observations along the equilibrium path (Sargent, 1999). To find an SCE is to solve a fixed-point problem. For our model, the solution to the SCE is to find the fixed point $\hat{\beta}$ that solves the orthogonality condition

$$
E\left[\hat{k}_{t}(\hat{\beta})-\hat{\beta}\right]=0
$$

where $E()$ is a mathematical unconditional expectation operator and $\hat{k}_{t}$ itself is a function of the belief $\hat{\beta}$ in self-confirming equilibrium such that

$$
\hat{k}_{t}(\hat{\beta})=\gamma_{1} \hat{\beta}+\gamma_{2} \hat{k}_{t-1}(\hat{\beta})+\gamma_{\nu 1} \hat{\nu}_{t}+\gamma_{\mu 1} \hat{\mu}_{t}+\gamma_{\nu 2} \hat{\nu}_{t-1}+\gamma_{\mu 2} \hat{\mu}_{t-1} \text {. }
$$


Proposition 3. As $g \rightarrow 0$, the belief sequence $\left\{\hat{\beta}_{t}\right\}$ in (32) converges weakly to the unique and stationary SCE given by $\hat{\beta}=0$ for all admissible values of the deep parameters.

Proof. From (33) one can see that $\hat{k}_{t}$ is a function of current and past beliefs and fundamental shocks. We denote this function as $\kappa()$ such that

$$
\hat{k}_{t}=\kappa\left(\hat{\beta}_{t}, \hat{\beta}_{t-1}, \ldots, \nu_{t}, \nu_{t-1}, \ldots, \mu_{t}, \mu_{t-1}, \ldots\right) .
$$

Denote

$$
\begin{aligned}
\tilde{\kappa}\left(\hat{\beta}_{t}, \hat{\beta}_{t-1}, \ldots, \nu_{t}, \nu_{t-1}, \ldots, \mu_{t}, \mu_{t-1}, \ldots\right) \\
\quad=\kappa\left(\hat{\beta}_{t}, \hat{\beta}_{t-1}, \ldots, \nu_{t}, \nu_{t-1}, \ldots, \mu_{t}, \mu_{t-1}, \ldots\right)-\hat{\beta}_{t}
\end{aligned}
$$

We can then rewrite the CGL algorithm (32) as

$$
\hat{\beta}_{t}=\hat{\beta}_{t-1}+g \tilde{\kappa}\left(\hat{\beta}_{t}, \hat{\beta}_{t-1}, \ldots, \nu_{t}, \nu_{t-1}, \ldots, \mu_{t}, \mu_{t-1}, \ldots\right) .
$$

To prove that (34) holds at $\hat{\beta}=0$ and the fixed point $\hat{\beta}=0$ is unique, we denote the left-hand-side term in (34) by

$$
G(\hat{\beta})=E \tilde{\kappa}\left(\hat{\beta}, \hat{\beta}, \ldots, \nu_{t}, \nu_{t-1}, \ldots, \mu_{t}, \mu_{t-1}, \ldots\right) .
$$

Under our assumptions, it follows from Kushner and Yin (1997) that as $g \rightarrow 0$, the beliefs $\hat{\beta}_{t}$ in (35) converge weakly to the solution of the ordinary differential equation (ODE)

$$
\dot{\hat{\beta}}=G(\hat{\beta})
$$

One can further show that

$$
G(\hat{\beta})=\left(\frac{\gamma_{2}+\gamma_{1}-1}{1-\gamma_{2}}\right) \hat{\beta} .
$$

Since $\gamma_{1}>0, \gamma_{2}>0$, and $\gamma_{2}+\gamma_{1}<1$, the ODE has a unique fixed point at $\hat{\beta}=0$. The ODE is stable since $\left(\gamma_{2}+\gamma_{1}-1\right) /\left(1-\gamma_{2}\right)<0$.

As one can see from Proposition 3, the SCE is exactly the same as the rational expectations steady state. Since an SCE is a limit of adaptive (learning) dynamics, it is important to characterize these dynamics and to study whether they are significantly different from dynamics under rational expectations. We rewrite the stochastic processes (32) and (33) as 


$$
\left[\begin{array}{l}
\hat{\beta}_{t} \\
\hat{k}_{t}
\end{array}\right]=\left[\begin{array}{cc}
1-g & g \\
(1-g) \gamma_{1} & \gamma_{2}+g \gamma_{1}
\end{array}\right]\left[\begin{array}{l}
\hat{\beta}_{t-1} \\
\hat{k}_{t-1}
\end{array}\right]+\left[\begin{array}{cc}
0 & 0 \\
\gamma_{\nu 1} & \gamma_{\mu 1}
\end{array}\right]\left[\begin{array}{l}
\hat{\nu}_{t} \\
\hat{\mu}_{t}
\end{array}\right]+\left[\begin{array}{cc}
0 & 0 \\
\gamma_{\nu 2} & \gamma_{\mu 2}
\end{array}\right]\left[\begin{array}{l}
\hat{\nu}_{t-1} \\
\hat{\mu}_{t-1}
\end{array}\right] .
$$

Given the initial belief $\hat{\beta}_{-1}$, the initial capital stock $\hat{k}_{-1}$, and the shock processes, the bivariate autoregressive process (36) determines the belief and capital dynamics jointly; then, (23)-(26) in Section III, or (A1)-(A4) in Appendix A, determine the dynamics of investment, labor, output, and consumption.

Proposition 4. The learning dynamics, described by (23)-(26) and (36) for $g \in(0,1)$, are stationary for all admissible values of the deep parameters.

Proof. Given (A1)-(A4) in Appendix A that characterize the dynamics of investment, labor, output, and consumption as a function of $\hat{k}_{t}$, it suffices to show that (36) is a stationary process. The two characteristic roots of the $2 \times 2$ coefficient matrix of $\hat{\beta}_{t-1}$ and $\hat{k}_{t-1}$ on the right-hand side of (36) are

$$
\begin{aligned}
& \lambda_{1}=\frac{\left(1-g+\gamma_{2}+g \gamma_{1}\right)-\sqrt{\left(1-g+\gamma_{2}+g \gamma_{1}\right)^{2}-4(1-g) \gamma_{2}}}{2}, \\
& \lambda_{2}=\frac{\left(1-g+\gamma_{2}+g \gamma_{1}\right)+\sqrt{\left(1-g+\gamma_{2}+g \gamma_{1}\right)^{2}-4(1-g) \gamma_{2}}}{2} .
\end{aligned}
$$

Since $\gamma_{1}>0, \gamma_{2}>0$, and $\gamma_{1}+\gamma_{2}<1$ for all admissible values of the deep parameters, it follows that both $\lambda_{1}$ and $\lambda_{2}$ are real numbers and for any $g \in(0,1), 0<\lambda_{1}<\lambda_{2}<1$. Hence, the adaptive process for $\left\{\hat{\beta}_{t}, \hat{k}_{t}\right\}$, given by (36), is stationary.

Proposition 4 implies that the learning dynamics studied in this paper remain in the domain of attraction of the SCE (the rational expectations steady state) and thus the probability of escapes from the SCE is very small.

To assess how different the learning dynamics differ from dynamics under rational expectations, we derive the belief and capital dynamics under the CGL as

$$
\begin{gathered}
\left(1-\lambda_{1} L\right)\left(1-\lambda_{2} L\right) \hat{\beta}_{t}=g\left(\gamma_{\nu 1} \hat{\nu}_{t-1}+\gamma_{\mu 1} \hat{\mu}_{t-1}+\gamma_{\nu 2} \hat{\nu}_{t-2}+\gamma_{\mu 2} \hat{\mu}_{t-2}\right), \\
\left(1-\lambda_{1} L\right)\left(1-\lambda_{2} L\right) \hat{k}_{t}=[1-(1-g) L]\left(\gamma_{\nu 1} \hat{\nu}_{t}+\gamma_{\mu 1} \hat{\mu}_{t}+\gamma_{\nu 2} \hat{\nu}_{t-1}+\gamma_{\mu 2} \hat{\mu}_{t-1}\right),
\end{gathered}
$$

where $L$ is the lag operator. It follows from (37) that 


$$
\begin{aligned}
\hat{\beta}_{t}=g \sum_{i=1}^{\infty} \sum_{j=1}^{i} \frac{\lambda_{1}^{j}-\lambda_{2}^{j}}{\lambda_{1}-\lambda_{2}}\left(\gamma_{\nu 1} \rho_{\nu}^{i-j} \varepsilon_{\nu, t-i}+\gamma_{\mu 1} \rho_{\mu}^{i-j} \varepsilon_{\mu, t-i}\right. & \\
& \left.+\gamma_{\nu 2} \rho_{\nu}^{i-j} \varepsilon_{\nu, t-1-i}+\gamma_{\mu 2} \rho_{\mu}^{i-j} \varepsilon_{\mu, t-1-i}\right) .
\end{aligned}
$$

This can be simplified to

$$
\begin{aligned}
\hat{\beta}_{t} & =g \gamma_{\nu 1} \varepsilon_{\nu, t-1}+g \sum_{i=2}^{\infty}\left[\gamma_{\nu 1} \frac{\lambda_{1}^{i}-\lambda_{2}^{i}}{\lambda_{1}-\lambda_{2}}+\left(\gamma_{\nu 1}+\gamma_{\nu 2} \rho_{\nu}^{-1}\right) \sum_{j=1}^{i-1} \frac{\lambda_{1}^{j}-\lambda_{2}^{j}}{\lambda_{1}-\lambda_{2}} \rho_{\nu}^{i-j}\right] \varepsilon_{\nu, t-i} \\
& +g \gamma_{\mu 1} \varepsilon_{\mu, t-1}+g \sum_{i=2}^{\infty}\left[\gamma_{\mu 1} \frac{\lambda_{1}^{i}-\lambda_{2}^{i}}{\lambda_{1}-\lambda_{2}}+\left(\gamma_{\mu 1}+\gamma_{\mu 2} \rho_{\mu}^{-1}\right) \sum_{j=1}^{i-1} \frac{\lambda_{1}^{j}-\lambda_{2}^{j}}{\lambda_{1}-\lambda_{2}} \rho_{\mu}^{i-j}\right] \varepsilon_{\mu, t-i} .
\end{aligned}
$$

If $\rho_{\nu} \neq \lambda_{1}$ or $\lambda_{2}$, and $\rho_{\mu} \neq \lambda_{1}$ or $\lambda_{2}$, then it simplifies further to

$$
\begin{aligned}
& \hat{\beta}_{t}=g \gamma_{\nu 1} \varepsilon_{\nu, t-1}+g \sum_{i=1}^{\infty} {\left[\gamma_{\nu 1} \frac{\lambda_{1}^{i+1}-\lambda_{2}^{i+1}}{\lambda_{1}-\lambda_{2}}\right.} \\
&\left.+\left(\rho_{\nu} \gamma_{\nu 1}+\gamma_{\nu 2}\right)\left(\frac{\lambda_{1}}{\lambda_{1}-\lambda_{2}} \frac{\lambda_{1}^{i}-\rho_{\nu}^{i}}{\lambda_{1}-\rho_{\nu}}-\frac{\lambda_{2}}{\lambda_{1}-\lambda_{2}} \frac{\lambda_{2}^{i}-\rho_{\nu}^{i}}{\lambda_{2}-\rho_{\nu}}\right)\right] \varepsilon_{\nu, t-1-i} \\
&+g \gamma_{\mu 1} \varepsilon_{\mu, t-1}+g \sum_{i=1}^{\infty}\left[\gamma_{\mu 1} \frac{\lambda_{1}^{i+1}-\lambda_{2}^{i+1}}{\lambda_{1}-\lambda_{2}}\right. \\
&\left.+\left(\rho_{\mu} \gamma_{\mu 1}+\gamma_{\mu 2}\right)\left(\frac{\lambda_{1}}{\lambda_{1}-\lambda_{2}} \frac{\lambda_{1}^{i}-\rho_{\mu}^{i}}{\lambda_{1}-\rho_{\mu}}-\frac{\lambda_{2}}{\lambda_{1}-\lambda_{2}} \frac{\lambda_{2}^{i}-\rho_{\mu}^{i}}{\lambda_{2}-\rho_{\mu}}\right)\right] \varepsilon_{\mu, t-1-i} .
\end{aligned}
$$

On the other hand, we can compute the rational expectations from (31) as

$$
\begin{aligned}
& E_{t} \hat{k}_{t+1}=\left[b\left(a+\rho_{\nu}\right)+d\right] \varepsilon_{\nu, t}+\left[c\left(a+\rho_{\mu}\right)+e\right] \varepsilon_{\mu, t} \\
& +\sum_{i=1}^{\infty}\left(b b_{i+1}+d b_{i}\right) \varepsilon_{\nu, t-i}+\sum_{i=1}^{\infty}\left(c c_{i+1}+e c_{i}\right) \varepsilon_{\mu, t-i} .
\end{aligned}
$$

A comparison of (40) and (41) shows that, although the SCE is the same as the steady state REE, the dynamics of beliefs $\hat{\beta}_{t}$ are both qualitatively and quantitatively different from those of expectations $E_{t} \hat{k}_{t+1}$. These differences lead to important differences in dynamics of other macroeconomic variables, as shown in the next section. 


\section{REE vs. SCE: Transmission of Technology Shocks}

We now analyze the transmission mechanisms of the model under both rational and adaptive expectations. We simulate the model using a few sets of parameter values, but the quantitative differences between learning and rational expectations dynamics exist for a wide range of values. The model parameters include $\beta$, the subjective discount factor; $\alpha$, the labor share of income; $\delta$, the capital depreciation rate; $\eta$, the inverse Frisch elasticity of labor supply; $\lambda_{z}$ and $\lambda_{q}$, the average growth rate of the neutral and biased technologies; $\xi$, the weight parameter in the preferences for leisure; $\rho_{\nu}, \rho_{\mu}, \sigma_{\nu}$, and $\sigma_{\mu}$, the parameters controlling the shock processes, and $g$, the constant gain in the learning process.

V.1. Benchmark parameterization. The benchmark parameter values we use for simulations, summarized in Table 1 , are based on quarterly frequency. We set $\alpha=$ 0.7 , corresponding to a labor income share of $70 \%$. We set $\lambda_{q}=1.008$ such that the investment-specific technology grows at an annual rate of $3.2 \%$, as suggested by Greenwood, Hercowitz, and Krusell (1997). We set $\lambda_{z}=1.0016$ such that, given our value of $\lambda_{q}$ and $\alpha$, real per capita GDP grows at an annual rate of $2 \%$ on the path of balanced growth. ${ }^{5}$ We set $\delta=0.03$ (corresponding to an annual depreciation rate of $12 \%$ ), a value used in business cycle studies. We set $\beta=0.99$. We set $\eta=2$, corresponding to a Frisch elasticity of 0.5(Pencavel, 1986). We set $\xi=34.22$ so that, given the values of other parameters, the steady-state hours worked are $30 \%$ of the time endowment (which is normalized to 1). For the parameters in the shock processes, we set $\rho_{\nu}=\rho_{\mu}=0.95$ and normalize the standard deviations such that $\sigma_{\nu}=\sigma_{\mu}=1$. Finally, we set the gain $g=0.05$ in the learning process.

V.2. Amplification effects. To understand the role of adaptive expectations in transmitting the two types of technology shocks, we compute impulse responses to both shocks.

V.2.1. Neutral technology shock. Figure 1 displays the impulse responses of aggregate variables to a neutral technology shock (normalized by one standard deviation) with our benchmark parameterization. The solid line represents the responses under

\footnotetext{
${ }^{5}$ The average growth rate for output in the model is given by $\lambda_{z} \lambda_{q}^{(1-\alpha) / \alpha}$.
} 
rational expectations and the dashed line represents the responses under adaptive expectations.

The responses of aggregate variables to a positive neutral technology shock in the rational expectations model should be familiar to a student of real business cycle studies. As the solid line in the figure shows, output rises on impact and declines gradually. Consumption, investment, hours, the real wage, and the real interest rate all co-move with output. In the impact period, consumption responds less and investment responds more than does output. These patterns of responses are consistent with the stylized facts about business cycles.

A well documented difficulty facing the standard RBC model with rational expectations lies in the labor market dynamics (Christiano and Eichenbaum, 1992). The RBC model typically fails to generate the observed large responses of labor hours and small responses of the real wage following a neutral technology shock. To understand this feature of the model, note that the shock raises the demand for labor at any given real wage, shifts the labor demand schedule out and creating a substitution effect. Thus, holding the labor supply schedule unchanged, the substitution effect drives up both hours and the real wage. In the mean time, since the shock is persistent and therefore raises future productivity, the associated wealth effect raises current consumption, which shifts the labor supply curve up. The wealth effect partially cancels out the substitution effect on hours, rendering the responses of equilibrium hours small; meanwhile, the wealth effect reinforces the substitution effect on the real wage, pushing up the equilibrium wage sharply. The lower the Frisch elasticity of labor supply, the greater the rise in the real wage. ${ }^{6}$ As is evident in the two lower panels of Figure 1 and the top panel in Table 2, the model with rational expectations implies that the initial response of hours is about $20 \%$ of that of output, while the magnitude of the real wage response about $80 \%$ of that of output response. Indeed, as shown in Table 2, in the rational expectations model, the cumulative responses of hours at all forecasting horizons (from 1 quarter through 24 quarters) are less than $20 \%$ and those of the real wages are more than $80 \%$ relative to the output responses.

Introducing learning helps alleviate some of the problems for the RBC model, especially for the labor market variables. The dashed lines in Figure 1 display the

\footnotetext{
${ }^{6}$ Even under the assumption of indivisible labor such that the aggregate labor supply elasticity is arbitrarily large (Hansen, 1985; Rogerson, 1988), the real wage still rises sharply.
} 
dynamic responses of the aggregate variables in the model with adaptive expectations following a positive neutral technology shock. We first note that the shock raises the demand for labor and, as in the rational expectations model, the substitution effect leads to a rise in both hours and the real wage. Unlike the rational expectations model, however, the wealth effect is muted because agents form expectations of the future productivity and income based on past observations. Since the wealth effect is muted, the initial rise in the real interest rate leads to an initial decline in consumption because of the intertemporal substitution effect. The decline in consumption in the impact period shifts the labor supply curve downward. Consequently, equilibrium hours rise sharply and the equilibrium real wage rises only modestly. The sharp rise in hours, along with the positive productivity shock, leads to a sharp rise in output; as consumption declines initially, the sharp rise in output leads to a sharp increase in investment. As shown in the lower panel of Table 2, the model with adaptive expectations implies that, at least in the short run, the cumulative responses of hours relative to output are much larger than those in the rational expectations model while the cumulative responses of the real wage relative to output are much smaller. In Section V.4,we will discuss the fluctuations of consumption relative to those of output.

In summary, following a neutral technology shock, introducing learning amplifies the response of hours and dampens the responses of the real wage. Furthermore, learning helps amplify the responses of other aggregate variables, including output, consumption, investment, and the real interest rate.

V.2.2. Biased technology shock. Although the effects of neutral technology shocks are well studied, the literature on the effects of investment-specific shocks is scarce, with the notable exceptions of Greenwood, Hercowitz, and Krusell (2000), Krusell, Ohanian, Ríos-Rull, and Violante (2000), Fisher (2006), and He and Liu (2008). To our best knowledge, there has been no published study that examines the effects of biased technology shocks (such as the investment-specific shocks) in the context of adaptive expectations.

In Figure 2, we plot the impulse responses following a positive biased technology shock for both the rational expectations and adaptive expectations models. In both models, the shock leads to a rise in output, investment, hours, and the real interest rate, but a short-run decline in consumption and the real wage. 
For the rational expectations model, as the biased shock raises the efficiency of investment, investment goods today become cheaper and current consumption becomes more expensive. This type of shock, unlike the neutral technology shock, shifts resources from consumption to investment. Consequently, investment rises and consumption declines for several periods. The decline in consumption shifts the labor supply curve down so much that the real wage declines somewhat while hours rise. The rise in labor hours helps produce more output. Meanwhile, as capital becomes more productive following the shock, the real interest rate increases.

In the learning model, because agents do not (rationally) foresee the increase in the future level of investment technology, they respond to the positive shock as though it had only a temporary effect. The muted channel of the wealth effect makes the intertemporal substitution effect stronger under learning than that under rational expectations. Consequently, the demand for current investment rises sharply, leading to a rise in investment and a fall in consumption in a magnitude more than that in the rational expectations model. The sharp decline in consumption amplifies the decline in the real wage and the rise in hours. The amplified increase in hours in turn leads to a sharp rise in output and thus in the real interest rate.

In summary, following a positive biased technology shock, the responses of all the aggregate variables are substantially amplified in the learning model, as compared to those under rational expectations. Overall, relaxing the assumption of perfect rationality helps give a larger role to both neutral and biased technology shocks in shaping business cycles.

Table 3 reports the cumulative responses of some macroeconomic variables relative to output responses at various forecasting horizons. Unlike the results for the neutral technology shock (Table 2), introducing learning does not change, at least in the short run (up to 4 quarters from the impact period), the relative fluctuations of consumption, investment, hours, and the real wage from the results implied by the rational expectations model. In longer horizons (8 quarters and beyond), however, the fluctuations of consumption, investment, hours, and the real wage, relative to those of output, are larger under learning than those under rational expectations.

V.2.3. An alternative learning rule. One important question is whether our main findings hinge on the particular learning rule (32). To address this issue, we consider 
a sophisticated learning rule that resembles the rational expectations solution (30). Specifically, we replace $E_{t} \hat{k}_{t+1}$ by $\hat{E}_{t} \hat{k}_{t+1}$ such that

$$
\hat{E}_{t} \hat{k}_{t+1}=x_{t}^{\prime} \alpha_{t+1 \mid t}
$$

where

$$
x_{t}=\left[\begin{array}{c}
\hat{k}_{t} \\
\hat{\nu}_{t} \\
\hat{\mu}_{t}
\end{array}\right] .
$$

Agents update their beliefs $\alpha_{t+1 \mid t}$ using the following adaptive learning algorithm:

$$
\begin{gathered}
\alpha_{t+1 \mid t}=\alpha_{t \mid t-1}+g H_{t} x_{t-1}\left(\hat{k}_{t}-x_{t-1}^{\prime} \alpha_{t \mid t-1}\right), \\
H_{t+1}^{-1}=H_{t}^{-1}+g\left(x_{t} x_{t}^{\prime}-H_{t}^{-1}\right) .
\end{gathered}
$$

This alternative learning mechanism ensures that the equilibrium under adaptive learning is E-stable in the sense of Evans and Honkapohja (2001). Because our alternative learning rule is separate from agents' decision making, the dynamic responses of aggregate variables under this new rule (not reported) are very similar to those under our benchmark learning rule in Sections V.2.1 and V.2.2. Such a similarity holds for a wide range of parameter values (including those studied later in this paper).

We have also experimented with other learning rules and the main results are insensitive to different learning mechanisms.

V.3. Relative importance of biased vs. neutral technology shocks. We have shown that learning amplifies the effects of both neutral and biased technology shocks. We now examine whether learning changes the relative importance of the two shocks.

In Table 4, we report these results for our benchmark model. For each variable, we first compute the contribution of the biased technology shock, relative to the neutral technology shock, in forecasting variances for both rational and adaptive expectations models. We then compute the ratio of such a contribution for the learning model to that for the rational expectations model. This ratio is reported in Table 4 . If the ratio is larger than one, it means that the relative contribution of the biased technology shock is more important under learning than under rational expectations. At the 4-quarter forecasting horizon, for instance, the biased technology shock accounts for only $6.06 \%$ of the output variance in the rational expectations model, while it accounts for $27.19 \%$ in the learning model (these values are not reported in the table). Thus, 
the contribution of the biased technology shock for the one-quarter ahead output variance in the learning model is 4.49 times that in the rational expectations model (this ratio is reported in the table).

As one can see from the table, introducing learning substantially amplifies the contribution of the biased technology shock relative to the neutral technology shock for output, consumption, and investment. Interestingly, the relative contribution of the biased technology shock for hours is the same across the two models.

V.4. Indivisible labor. In Table 2, we have seen that learning makes consumption fluctuate more than output. In this section, we show that this result is sensitive to parameter values and, in particular, to the value of the Frisch elasticity of labor supply.

Consider the case where all the benchmark parameter values are the same except for the inverse Frisch elasticity parameter $\eta$, which we set to zero. The zero value of $\eta$ corresponds to indivisible labor in the sense of Hansen (1985) and Rogerson (1988) and implies an infinite labor supply elasticity at the aggregate level.

Tables 5 and 6 display the cumulative responses of several macroeconomic variables relative to those of output for $\eta=0$, with all the other parameters the same as their benchmark values. Table 5 shows that, following the neutral technology shock, the model with rational expectations (with $\eta=0$ ) generates larger responses of hours and smaller responses of the real wage than those in the benchmark model, at least in the short run. Since consumption is proportional to the real wage (labor productivity) when $\eta=0$ (see $(9)$ ), the responses of consumption are smaller than those in the benchmark model (compared to Table 2). In the learning model, the responses of consumption relative to those of output become much smaller with indivisible labor (Table 5) than those with the benchmark value of labor elasticity (Table 2), while the relative fluctuations of hours are larger and the relative fluctuations of the real wage are smaller when compared to the benchmark case.

Table 6 reports the cumulative responses of consumption, investment, hours, and the real wage relative to those of output for $\eta=0$, following a positive biased technology shock. The table shows that learning does not change the relative fluctuations of these variables in the short run, but amplifies the fluctuations of these variables at the longer horizon (8 quarters and beyond). 
Table 7 reports the relative contribution of the biased technology shock. As in the benchmark case, the biased technology becomes more important under learning than under rational expectations, but the magnitude of the relative importance of the biased technology shock becomes smaller than that in the benchmark model where the the Frisch elasticity of labor is smaller.

V.5. Less persistent shocks. Since the transmission of the shocks in the model with adaptive expectations works through the muted wealth effect, the quantitative importance of learning should depend on the persistence of the shock. To understand to what extent the propagation mechanism in the learning model depends on the persistence of the shocks, we consider the case with less persistent shocks. In particular, we set $\rho_{\nu}=\rho_{\mu}=0.7$ (instead of 0.95 ) and report the impulse responses following each type of technology shocks for the two alternative models.

Figure 3 displays the impulse responses to a positive neutral technology shocks under rational expectations (the solid lines) and under adaptive expectations (the dashed lines). Since the shock is less persistent, the wealth effect is weaker so that, in the rational expectations model, the rise in consumption is smaller and the rise in hours is larger than that under the benchmark parameterization (in comparison with Figure 1). For the same reason (i.e., the wealth effect is weaker with less persistent shocks), introducing adaptive learning dampens the response of the real wage and amplifies the responses of other aggregate variables, but to a lesser extent than in the benchmark case shown in Figure 1. Moreover, the responses of both consumption and the real wage display a clear hump shape; the responses of investment, hours, and the real interest rate all display an inverted hump shape; and output rises in the impact period and declines monotonically thereafter. Under adaptive expectations, the representative agent is backward looking when forming expectations. In the impact period, the wealth effect of the shock is muted; the intertemporal substitution effect induced by the rise in the real interest rate makes consumption more expensive and saving more attractive. Thus, in the short run, consumption falls and investment rises. Overtime, however, the agent learns about the wealth effect of the positive technology shock while the intertemporal substitution effect becomes weaker as the real interest rate goes back to its steady state. Thus, consumption rises above the steady state and keeps rising before it begins to decline back to the steady state. 
The rise in consumption shifts the labor supply curve up and thus lowers labor hours and raises the real wage. As consumption climbs to its peak over time, hours and investment fall to the trough and the real wage rises to the peak. Since output falls back to the steady state over time, consumption, investment, and hours return gradually to the steady state.

Figure 4 displays the impulse responses to a positive biased technology shock in both the rational expectations model (the solid lines) and the adaptive expectations model (the dashed lines). As in the benchmark case (Figure 2), the responses in the adaptive expectations model are more pronounced than those in the rational expectations model, but the amplification effects of learning are smaller here with the less persistent shock. Similar to the case with neutral technology shock (Figure 3 ), the responses of consumption and the real wage both display a hump shape; the responses of investment, labor hours, and the real interest rate all display an inverted hump shape; and the response of output does not have a hump.

In summary, with less persistent shocks, the wealth effect in the rational expectations model becomes weaker and accordingly the amplification effect of adaptive expectations become weaker as well. The adaptive expectations model generates more pronounced hump-shaped responses while the rational expectations model does not.

V.6. Procyclical consumption responses. In Figure 1 (the benchmark case), we have seen that learning implies negative short-run responses of consumption following a positive neutral technology shock. For less persistent neutral shocks, the short-run responses of consumption remain negative, although to a lesser extent, as shown in Figure 3. We now show that this problem can be alleviated when we combine less persistent shocks with indivisible labor.

Figures 5 and 6 display the impulse responses with indivisible labor $(\eta=0)$ and with low persistence in the shocks $\left(\rho_{\nu}=\rho_{\mu}=0.7\right)$, while all other parameters are kept at their benchmark values. As we have discussed in Sections V.4 and V.5, these parameter values imply a smaller wealth effect under rational expectations than that in the benchmark model. As in the benchmark model, learning here still mitigates the wealth effect, but to a lesser extent. Indeed, under this new parameter configuration, learning no longer produces a sharp short-run decline in consumption 
following the positive neutral technology shock. Figure 5 shows that, in contrast to the benchmark results reported in Figure 1, the impact effect of the neutral shock on consumption becomes slightly positive and the subsequent responses of consumption are procyclical.

As can be seen in Figures 5 and 6, our general conclusion remains the same under this new parameter configuration: learning amplifies the dynamic responses of all aggregate variables to both technology shocks and improves the model's predictions on the labor market dynamics.

\section{Conclusion}

We have studied a standard growth model with adaptive expectations in which beliefs are decoupled from decision rules. We have proven that there exists a unique, stable SCE in our learning model and that the SCE is the same as the steady state REE. In contrast to the existing literature, however, we have shown that the learning model can generate substantially different dynamics from those implied by the rational expectations model. These differences are not driven by escape dynamics.

It is known that technology shocks in the standard growth model do not generate enough fluctuations in key macroeconomic variables such as hours and output. Introducing misspecified learning in the growth model dampens the wealth effect. This muted wealth effect, coupled with the strong intertemporal substitution effect, amplifies the responses of macroeconomic variables and can make dynamic responses hump-shaped. In summary, the learning model gives technology shocks a much more prominent role in causing fluctuations of macroeconomic variables than does the rational expectations model. 
The coefficients in (29) in Proposition 1 are defined as:

$$
\begin{gathered}
\gamma_{1, \mathrm{com}}=\beta(1-\alpha)\left(c_{k}+1\right)\left[1+(1+\eta) c_{k}\right]+\eta\left(1-\alpha i_{k}\right)+(1-\alpha+\eta), \\
\gamma_{2, \mathrm{com}}=\left[\beta(1-\alpha)\left(c_{k}+1\right)-1\right]\left[(1+\eta) c_{k}+1\right]+(1+\eta)\left(1-\alpha i_{k}\right), \\
\gamma_{1}=\frac{\beta(1-\alpha)\left(c_{k}+1\right)+\eta}{\gamma_{1, \mathrm{com}}}, \\
\gamma_{2}=\frac{(1-\alpha)(1+\eta) y_{k}+(1-\alpha+\eta)\left(1-i_{k}\right)}{\gamma_{1, \mathrm{com}}} \\
\gamma_{\nu 1}=\frac{\left(\rho_{\nu}-1\right) \gamma_{2, \mathrm{com}}+\alpha \eta y_{k}-(1-\alpha+\eta)\left(c_{k}+1\right)}{\gamma_{1, \mathrm{com}}} \\
\gamma_{\mu 1}=\frac{\left(\rho_{\mu}-1\right) \gamma_{2, \mathrm{com}}+\alpha \eta y_{k}-(1-\alpha+\eta)\left(c_{k}+1\right)}{\alpha \gamma_{1, \mathrm{com}}} \\
\gamma_{1, \mathrm{com}} \\
\gamma_{\nu 2}=\frac{(1-\alpha+\eta)\left(c_{k}+1\right)-\alpha \eta y_{k}}{\alpha \gamma_{1, \mathrm{com}}} \\
\gamma_{\mu 2}=\frac{(1-\alpha+\eta)\left(c_{k}+1\right)-\alpha \eta y_{k}}{}
\end{gathered}
$$

The steady state ratios such as $c_{k}$ and $i_{k}$ have been derived in Section III. One can verify that, for all admissible values of the deep parameters, that is, for any $\beta \in(0,1), \eta \geq 0, \alpha \in(0,1), \delta \in[0,1], \lambda_{z} \geq 1$, and $\lambda_{q} \geq 1$, all the steady-state ratios are well-defined and positive, and so are $\gamma_{1}$ and $\gamma_{2}$.

The closed-form solutions for investment, hours, output, and consumption are derived as the the following system of equations under either rational or adaptive expectations:

$$
\begin{gathered}
\hat{i}_{t}=k_{i} \hat{k}_{t}+\left(1-k_{i}\right) \hat{k}_{t-1}+\left(k_{i}-1\right) \Delta \hat{\nu}_{t}+\left(\frac{k_{i}-1}{\alpha}\right) \Delta \hat{\mu}_{t}, \\
\hat{l}_{t}=\frac{1}{\left[(1+\eta) c_{k}+\alpha i_{k}\right]} \hat{k}_{t}-\frac{\left[1-\alpha i_{k}\right]}{\left[(1+\eta) c_{k}+\alpha i_{k}\right]} \hat{k}_{t-1} \\
+\frac{\left(1-\alpha i_{k}\right)}{\left[(1+\eta) c_{k}+\alpha i_{k}\right]} \Delta \hat{\nu}_{t}+\frac{\left(\alpha^{-1}-i_{k}\right)}{\left[(1+\eta) c_{k}+\alpha i_{k}\right]} \Delta \hat{\mu}_{t},
\end{gathered}
$$




$$
\begin{gathered}
\hat{y}_{t}=\frac{\alpha}{\left[(1+\eta) c_{k}+\alpha i_{k}\right]} \hat{k}_{t}+\frac{\left[(1-\alpha)(1+\eta) c_{k}-\alpha\left(1-i_{k}\right)\right]}{\left[(1+\eta) c_{k}+\alpha i_{k}\right]} \hat{k}_{t-1} \\
+\frac{\alpha\left(1-i_{k}\right)-(1-\alpha)(1+\eta) c_{k}}{\left[(1+\eta) c_{k}+\alpha i_{k}\right]} \Delta \hat{\nu}_{t} \\
+\frac{\left(1-i_{k}\right)-\alpha^{-1}(1-\alpha)(1+\eta) c_{k}}{\left[(1+\eta) c_{k}+\alpha i_{k}\right]} \Delta \hat{\mu}_{t} \\
\hat{c}_{t}=\frac{\alpha-1-\eta}{\left[(1+\eta) c_{k}+\alpha i_{k}\right]} \hat{k}_{t}+\frac{\left[(1-\alpha)(1+\eta) y_{k}+(1+\eta-\alpha)\left(1-i_{k}\right)\right]}{\left[(1+\eta) c_{k}+\alpha i_{k}\right]} \hat{k}_{t-1} \\
+\frac{\alpha\left(1+\eta i_{k}\right)-(1+\eta)\left[1+(1-\alpha) c_{k}\right]}{\left[(1+\eta) c_{k}+\alpha i_{k}\right]} \Delta \hat{\nu}_{t} \\
+\frac{\left(1+\eta i_{k}\right)-\alpha^{-1}(1+\eta)\left[1+(1-\alpha) c_{k}\right]}{\left[(1+\eta) c_{k}+\alpha i_{k}\right]} \Delta \hat{\mu}_{t} .
\end{gathered}
$$

It is clear how the equilibrium can be solved. Once the solution for capital is obtained, as shown in Section IV, Equation (A1) can be used to solve for investment, (A2) for labor, (A3) for output, and (A4) for consumption.

\section{Appendix B. Proof of Proposition 1}

By successive substitutions in (23)-(27), one can derive (29). Specific steps are described below.

We begin by first deriving the following two relations from (25) and (26):

$$
\begin{aligned}
& \hat{y}_{t}=\hat{i}_{t}-(1+\eta) c_{i} \hat{l}_{t}, \\
& \hat{c}_{t}=\hat{i}_{t}-(1+\eta) y_{i} \hat{l}_{t} .
\end{aligned}
$$

Substituting (A5) into (23), we get:

$$
\hat{i}_{t}=\left[(1+\eta) c_{i}+\alpha\right] \hat{l}_{t}+(1-\alpha) \hat{k}_{t-1}-(1-\alpha) \Delta \hat{\nu}_{t}-\left(\frac{1-\alpha}{\alpha}\right) \Delta \hat{\mu}_{t}
$$

Substituting (A7) into (24) yields

$$
\hat{k}_{t}=\left[(1+\eta) c_{k}+\alpha i_{k}\right] \hat{l}_{t}+\left(1-\alpha i_{k}\right) \hat{k}_{t-1}-\left(1-\alpha i_{k}\right) \Delta \hat{\nu}_{t}-\left(\frac{1-\alpha i_{k}}{\alpha}\right) \Delta \hat{\mu}_{t} .
$$


Substituting (A6) and (A7) into (27) yields

$$
\begin{aligned}
{\left[1-\frac{\alpha \beta(1-\delta)}{\lambda_{z} \lambda_{q}^{1 / \alpha}}+\eta\right] } & E_{t} \hat{l}_{t+1}-\left[1-\frac{\alpha \beta(1-\delta)}{\left.\lambda_{z} \lambda_{q}^{1 / \alpha}\right] \hat{k}_{t}}\right. \\
& =(1-\alpha+\eta) \hat{l}_{t}-(1-\alpha) \hat{k}_{t-1} \\
& +(1-\alpha) \Delta \hat{\nu}_{t}+\frac{\alpha \beta(1-\delta)}{\lambda_{z} \lambda_{q}^{1 / \alpha}} E_{t} \Delta \hat{\nu}_{t+1} \\
& +\frac{1-\alpha}{\alpha} \Delta \hat{\mu}_{t}+\frac{\beta(1-\delta)}{\lambda_{z} \lambda_{q}^{1 / \alpha}} E_{t} \Delta \hat{\mu}_{t+1} .
\end{aligned}
$$

Rewrite (A8) as

$$
\begin{aligned}
& \hat{l}_{t}=\frac{1}{(1+\eta) c_{k}+\alpha i_{k}} \hat{k}_{t} \\
& -\frac{1-\alpha i_{k}}{(1+\eta) c_{k}+\alpha i_{k}} \hat{k}_{t-1} \\
& +\frac{1-\alpha i_{k}}{(1+\eta) c_{k}+\alpha i_{k}} \Delta \hat{\nu}_{t} \\
& +\frac{\left\{1-\alpha i_{k}\right\}\left(\frac{1}{\alpha}\right)}{(1+\eta) c_{k}+\alpha i_{k}} \Delta \hat{\mu}_{t} .
\end{aligned}
$$

It follows that

$$
\begin{aligned}
& {\left[1-\frac{\alpha \beta(1-\delta)}{\lambda_{z} \lambda_{q}^{1 / \alpha}}+\eta\right] E_{t} \hat{l}_{t+1}=\frac{\left[1-\frac{\alpha \beta(1-\delta)}{\lambda_{z} \lambda_{q}^{1 / \alpha}}+\eta\right]}{\left(1-\frac{1-\delta}{\lambda_{z} \lambda_{q}^{1 / \alpha}}\right)\left(\frac{1-c_{y}}{i_{y}}\right)\left[\frac{c_{y}(1+\eta)}{1-c_{y}}+\alpha\right]} E_{t} \hat{k}_{t+1}} \\
& -\frac{\left[1-\frac{\alpha \beta(1-\delta)}{\lambda_{z} \lambda_{q}^{1 / \alpha}}+\eta\right]\left\{1-\alpha i_{k}\right\}}{\left(1-\frac{1-\delta}{\lambda_{z} \lambda_{q}^{1 / \alpha}}\right)\left(\frac{1-c_{y}}{i_{y}}\right)\left[\frac{c_{y}(1+\eta)}{1-c_{y}}+\alpha\right]} \hat{k}_{t} \\
& +\frac{\left[1-\frac{\alpha \beta(1-\delta)}{\lambda_{z} \lambda_{q}^{1 / \alpha}}+\eta\right]\left\{\left(1-\frac{1-\delta}{\lambda_{z} \lambda_{q}^{1 / \alpha}}\right)\left[\frac{\left(1-c_{y}\right)(1-\alpha)}{i_{y}}\right]+\frac{1-\delta}{\lambda_{z} \lambda_{q}^{1 / \alpha}}\right\}}{\left(1-\frac{1-\delta}{\lambda_{z} \lambda_{q}^{1 / \alpha}}\right)\left(\frac{1-c_{y}}{i_{y}}\right)\left[\frac{c_{y}(1+\eta)}{1-c_{y}}+\alpha\right]} E_{t} \Delta \hat{\nu}_{t+1} \\
& +\frac{\left[1-\frac{\alpha \beta(1-\delta)}{\lambda_{z} \lambda_{q}^{1 / \alpha}}+\eta\right]\left\{\left(1-\frac{1-\delta}{\lambda_{z} \lambda_{q}^{1 / \alpha}}\right)\left[\frac{\left(1-c_{y}\right)(1-\alpha)}{i_{y}}\right]+\frac{1-\delta}{\lambda_{z} \lambda_{q}^{1 / \alpha}}\right\}\left(\frac{1}{\alpha}\right)}{\left(1-\frac{1-\delta}{\lambda_{z} \lambda_{q}^{1 / \alpha}}\right)\left(\frac{1-c_{y}}{i_{y}}\right)\left[\frac{c_{y}(1+\eta)}{1-c_{y}}+\alpha\right]} E_{t} \Delta \hat{\mu}_{t+1}
\end{aligned}
$$

Substituting (A10) and (A11) into (A9), and rearranging, we get

$\chi_{k, 1} E_{t} \hat{k}_{t+1}+\chi_{k, 0} \hat{k}_{t}+\chi_{k,-1} \hat{k}_{t-1}+\chi_{\nu, 1} E_{t} \Delta \hat{\nu}_{t+1}$

$$
+\chi_{\nu, 0} \Delta \hat{\nu}_{t}+\chi_{\mu, 1} E_{t} \Delta \hat{\mu}_{t+1}+\chi_{\mu, 0} \Delta \hat{\mu}_{t}=0,
$$


where

$$
\begin{aligned}
& \chi_{k, 1}=\frac{\left[1-\frac{\alpha \beta(1-\delta)}{\lambda_{z} \lambda_{q}^{1 / \alpha}}+\eta\right]}{\left(1-\frac{1-\delta}{\lambda_{z} \lambda_{q}^{1 / \alpha}}\right)\left(\frac{1-c_{y}}{i_{y}}\right)\left[\frac{c_{y}(1+\eta)}{1-c_{y}}+\alpha\right]} \\
& \chi_{k, 0}=-\frac{\left[1-\frac{\alpha \beta(1-\delta)}{\lambda_{z} \lambda_{q}^{1 / \alpha}}+\eta\right]\left\{1-\alpha i_{k}\right\}+(1-\alpha+\eta)}{\left(1-\frac{1-\delta}{\lambda_{z} \lambda_{q}^{1 / \alpha}}\right)\left(\frac{1-c_{y}}{i_{y}}\right)\left[\frac{c_{y}(1+\eta)}{1-c_{y}}+\alpha\right]}-\left[1-\frac{\alpha \beta(1-\delta)}{\lambda_{z} \lambda_{q}^{1 / \alpha}}\right] \\
& \chi_{k,-1}=\frac{(1-\alpha+\eta)\left\{1-\alpha i_{k}\right\}}{\left(1-\frac{1-\delta}{\lambda_{z} \lambda_{q}^{1 / \alpha}}\right)\left(\frac{1-c_{y}}{i_{y}}\right)\left[\frac{c_{y}(1+\eta)}{1-c_{y}}+\alpha\right]}+(1-\alpha) \\
& \chi_{\nu, 1}=\frac{\left[1-\frac{\alpha \beta(1-\delta)}{\lambda_{z} \lambda_{q}^{1 / \alpha}}+\eta\right]\left\{\left(1-\frac{1-\delta}{\lambda_{z} \lambda_{q}^{1 / \alpha}}\right)\left[\frac{\left(1-c_{y}\right)(1-\alpha)}{i_{y}}\right]+\frac{1-\delta}{\left.\lambda_{z} \lambda_{q}^{1 / \alpha}\right\}}\right.}{\left(1-\frac{1-\delta}{\lambda_{z} \lambda_{q}^{1 / \alpha}}\right)\left(\frac{1-c_{y}}{i_{y}}\right)\left[\frac{c_{y}(1+\eta)}{1-c_{y}}+\alpha\right]}-\frac{\alpha \beta(1-\delta)}{\lambda_{z} \lambda_{q}^{1 / \alpha}} \\
& \chi_{\nu, 0}=-\frac{(1-\alpha+\eta)\left\{\left(1-\frac{1-\delta}{\lambda_{z} \lambda_{q}^{1 / \alpha}}\right)\left[\frac{\left(1-c_{y}\right)(1-\alpha)}{i_{y}}\right]+\frac{1-\delta}{\lambda_{z} \lambda_{q}^{1 / \alpha}}\right\}}{\left(1-\frac{1-\delta}{\lambda_{z} \lambda_{q}^{1 / \alpha}}\right)\left(\frac{1-c_{y}}{i_{y}}\right)\left[\frac{c_{y}(1+\eta)}{1-c_{y}}+\alpha\right]}-(1-\alpha) \\
& \chi_{\mu, 1}=\frac{\left[1-\frac{\alpha \beta(1-\delta)}{\lambda_{z} \lambda_{q}^{1 / \alpha}}+\eta\right]\left\{\left(1-\frac{1-\delta}{\lambda_{z} \lambda_{q}^{1 / \alpha}}\right)\left[\frac{\left(1-c_{y}\right)(1-\alpha)}{i_{y}}\right]+\frac{1-\delta}{\lambda_{z} \lambda_{q}^{1 / \alpha}}\right\}\left(\frac{1}{\alpha}\right)}{\left(1-\frac{1-\delta}{\lambda_{z} \lambda_{q}^{1 / \alpha}}\right)\left(\frac{1-c_{y}}{i_{y}}\right)\left[\frac{c_{y}(1+\eta)}{1-c_{y}}+\alpha\right]}-\frac{\beta(1-\delta)}{\lambda_{z} \lambda_{q}^{1 / \alpha}} \\
& \chi_{\mu, 0}=-\frac{(1-\alpha+\eta)\left\{\left(1-\frac{1-\delta}{\lambda_{z} \lambda_{q}^{1 / \alpha}}\right)\left[\frac{\left(1-c_{y}\right)(1-\alpha)}{i_{y}}\right]+\frac{1-\delta}{\lambda_{z} \lambda_{q}^{1 / \alpha}}\right\}\left(\frac{1}{\alpha}\right)}{\left(1-\frac{1-\delta}{\lambda_{z} \lambda_{q}^{1 / \alpha}}\right)\left(\frac{1-c_{y}}{i_{y}}\right)\left[\frac{c_{y}(1+\eta)}{1-c_{y}}+\alpha\right]}-\frac{1-\alpha}{\alpha} .
\end{aligned}
$$

Further simplifying, we get

$$
\hat{k}_{t}=\chi_{k, 1}^{k} E_{t} \hat{k}_{t+1}+\chi_{k,-1}^{k} \hat{k}_{t-1}+\chi_{\nu, 1}^{k} E_{t} \Delta \hat{\nu}_{t+1}+\chi_{\nu, 0}^{k} \Delta \hat{\nu}_{t}+\chi_{\mu, 1}^{k} E_{t} \Delta \hat{\mu}_{t+1}+\chi_{\mu, 0}^{k} \Delta \hat{\mu}_{t},
$$

where

$$
\begin{gathered}
\chi_{k, 1}^{k}=\frac{\beta(1-\alpha)\left(c_{k}+1\right)+\eta}{\beta(1-\alpha)\left(c_{k}+1\right)\left[1+(1+\eta) c_{k}\right]+\eta\left(1-\alpha i_{k}\right)+(1-\alpha+\eta)} \\
\chi_{k,-1}^{k}=\frac{(1-\alpha)(1+\eta) y_{k}+(1-\alpha+\eta)\left(1-i_{k}\right)}{\beta(1-\alpha)\left(c_{k}+1\right)\left[1+(1+\eta) c_{k}\right]+\eta\left(1-\alpha i_{k}\right)+(1-\alpha+\eta)} \\
\chi_{\nu, 1}^{k}=\frac{\left[\beta(1-\alpha)\left(c_{k}+1\right)-1\right]\left[(1+\eta) c_{k}+1\right]+(1+\eta)\left(1-\alpha i_{k}\right)}{\beta(1-\alpha)\left(c_{k}+1\right)\left[1+(1+\eta) c_{k}\right]+\eta\left(1-\alpha i_{k}\right)+(1-\alpha+\eta)} \\
\chi_{\nu, 0}^{k}=-\frac{(1-\alpha+\eta)\left(c_{k}+1\right)-\alpha \eta y_{k}}{\beta(1-\alpha)\left(c_{k}+1\right)\left[1+(1+\eta) c_{k}\right]+\eta\left(1-\alpha i_{k}\right)+(1-\alpha+\eta)} \\
\chi_{\mu, 1}^{k}=\frac{\left[\beta(1-\alpha)\left(c_{k}+1\right)-1\right]\left[(1+\eta) c_{k}+1\right]+(1+\eta)\left(1-\alpha i_{k}\right)}{\beta(1-\alpha)\left(c_{k}+1\right)\left[1+(1+\eta) c_{k}\right]+\eta\left(1-\alpha i_{k}\right)+(1-\alpha+\eta)}\left(\frac{1}{\alpha}\right)
\end{gathered}
$$




$$
\chi_{\mu, 0}^{k}=-\frac{(1-\alpha+\eta)\left(c_{k}+1\right)-\alpha \eta y_{k}}{\beta(1-\alpha)\left(c_{k}+1\right)\left[1+(1+\eta) c_{k}\right]+\eta\left(1-\alpha i_{k}\right)+(1-\alpha+\eta)}\left(\frac{1}{\alpha}\right) .
$$

Simplifying further, we have

$$
\begin{aligned}
\hat{k}_{t}=\chi_{k, 1}^{k} E_{t} \hat{k}_{t+1}+\chi_{k,-1}^{k} \hat{k}_{t-1}-\chi_{\nu, 0}^{k} \hat{\nu}_{t-1}+\left[\left(\rho_{\nu}-1\right) \chi_{\nu, 1}^{k}+\chi_{\nu, 0}^{k}\right] \hat{\nu}_{t} & \\
& -\chi_{\mu, 0}^{k} \hat{\mu}_{t-1}+\left[\left(\rho_{\mu}-1\right) \chi_{\mu, 1}^{k}+\chi_{\mu, 0}^{k}\right] \hat{\mu}_{t},
\end{aligned}
$$

which gives the results in Appendix A.

\section{Appendix C. Proof of Proposition 2}

Because (29) is a second-order differential equation, there are only two solutions. We will show, next, that one solution is stationary and the other explosive. Thus, there is a unique stationary solution.

The coefficient $a$ in (30) takes on one of the following two values:

$$
a_{1}=\frac{1-\sqrt{1-4 \gamma_{1} \gamma_{2}}}{2 \gamma_{1}}, \quad a_{2}=\frac{1+\sqrt{1-4 \gamma_{1} \gamma_{2}}}{2 \gamma_{1}} .
$$

We can verify that $\gamma_{1}>0$ and $\gamma_{2}>0$ for all admissible values of the deep parameters. We can further show that $\gamma_{1}+\gamma_{2}<1$ if and only if $\beta(1-\delta)<\lambda_{z} \lambda_{q}^{1 / \alpha}$, which holds too for all admissible values of the deep parameters.

Since $\gamma_{1}>0, \gamma_{2}>0, \gamma_{1}+\gamma_{2}<1$, we have $\gamma_{1} \in(0,1), \gamma_{2} \in(0,1)$, and $4 \gamma_{1} \gamma_{2}<1$. It follows that $a_{1}$ and $a_{2}$ are real numbers. Knowing the above ranges for $\gamma_{1}$ and $\gamma_{2}$, we can in fact show that $a_{1} \in(0,1)$ and $a_{2}>1$. We can then verify that $\left(\rho_{\nu}+a_{1}\right) \gamma_{1}<1$ and $\left(\rho_{\mu}+a_{1}\right) \gamma_{1}<1$, which imply that $\gamma_{1} a_{1}<1$, and so the solution prescribed by $a=a_{1}$ above corresponds to a (unique) stationary rational expectations equilibrium. ${ }^{7}$ Given the initial condition $\hat{k}_{-1}$ and the driving processes, (30) completely pins down capital, and then (A1), (A2), (A3), and (A4) determine investment, labor, output, and consumption, respectively. From now on, whenever we mention REE, we refer to this stationary REE, where we also write $a_{1}$ simply as $a$.

\footnotetext{
${ }^{7}$ We can also show that, provided $\rho_{\nu} \neq a_{1}$ and $\rho_{\mu} \neq a_{1}$, the solution prescribed by $a=a_{2}$ above corresponds to an explosive path.
} 
TABLE 1. Parameter values

\begin{tabular}{llll}
\hline \hline Preference & $\beta=0.99$ & $\eta=2.0$ & $\xi$ adjusted \\
Labor share & $\alpha=0.7$ & & \\
Capital Depreciation & $\delta=0.03$ & & \\
Neutral Technology & $\lambda_{z}=1.0016$ & $\rho_{\nu}=0.95$ & $\sigma_{\nu}=1$ \\
Biased Technology & $\lambda_{q}=1.008$ & $\rho_{\mu}=0.95$ & $\sigma_{\mu}=1$ \\
Learning Gain & $g=0.05$ & & \\
\hline
\end{tabular}

TABLE 2. Cumulative responses relative to output following the neutral technology shock: benchmark parameters

\begin{tabular}{lllll}
\hline \hline \multicolumn{5}{c}{ Rational expectations model } \\
\hline Forecast Horizon & Consumption & Investment & Hours & Real wage \\
1 quarter & 0.39 & 2.89 & 0.20 & 0.80 \\
4 quarters & 0.47 & 2.66 & 0.18 & 0.82 \\
8 quarters & 0.55 & 2.40 & 0.15 & 0.85 \\
16 quarters & 0.67 & 2.03 & 0.11 & 0.89 \\
24 quarters & 0.75 & 1.78 & 0.09 & 0.92 \\
\hline \multicolumn{5}{c}{ Adaptive expectations model } \\
\hline Forecast Horizon & Consumption & Investment & Hours & Real wage \\
1 quarter & 1.19 & 7.85 & 0.73 & 0.27 \\
4 quarters & 0.73 & 4.60 & 0.38 & 0.62 \\
8 quarters & 0.95 & 2.88 & 0.27 & 0.80 \\
16 quarters & 1.11 & 1.93 & 0.22 & 0.92 \\
24 quarters & 1.17 & 1.63 & 0.21 & 0.97 \\
\hline
\end{tabular}


TABLE 3. Cumulative responses relative to output following the biased technology shock: benchmark parameters

\begin{tabular}{lllll}
\hline \hline \multicolumn{5}{c}{ Rational expectations model } \\
\hline Forecast Horizon & Consumption & Investment & Hours & Real wage \\
1 quarter & 3.29 & 14.39 & 1.43 & 0.43 \\
4 quarters & 2.01 & 10.39 & 1.00 & 0.23 \\
8 quarters & 1.09 & 7.53 & 0.70 & 0.40 \\
16 quarters & 0.75 & 4.81 & 0.41 & 0.64 \\
24 quarters & 0.83 & 3.54 & 0.28 & 0.76 \\
\hline \multicolumn{5}{c}{ Adaptive expectations model } \\
\hline Forecast Horizon & Consumption & Investment & Hours & Real wage \\
1 quarter & 3.29 & 14.39 & 1.43 & 0.43 \\
4 quarters & 2.07 & 9.97 & 0.96 & 0.40 \\
8 quarters & 2.09 & 7.85 & 0.79 & 0.68 \\
16 quarters & 2.28 & 6.93 & 0.74 & 0.93 \\
24 quarters & 2.38 & 6.66 & 0.72 & 1.04 \\
\hline
\end{tabular}

TABLE 4. Relative contribution of biased technology shocks with benchmark parameters: ratio of learning to $\mathrm{RE}$

\begin{tabular}{lllll}
\hline \hline Forecast Horizon & Output & Consumption & Investment & Hours \\
1 quarter & 8.87 & 1.08 & 1.28 & 1.00 \\
4 quarters & 4.49 & 1.42 & 1.28 & 1.00 \\
8 quarters & 2.65 & 1.95 & 1.31 & 1.00 \\
16 quarters & 1.57 & 2.72 & 1.36 & 1.00 \\
24 quarters & 1.24 & 2.46 & 1.38 & 1.00 \\
\hline
\end{tabular}


TABLE 5. Cumulative responses relative to output following the neutral technology shock: indivisible labor $(\eta=0)$

\begin{tabular}{lllll}
\hline \hline \multicolumn{5}{c}{ Rational expectations model } \\
\hline Forecast Horizon & Consumption & Investment & Hours & Real wage \\
1 quarter & 0.33 & 3.10 & 0.67 & 0.33 \\
4 quarters & 0.42 & 2.83 & 0.58 & 0.42 \\
8 quarters & 0.51 & 2.52 & 0.49 & 0.51 \\
16 quarters & 0.65 & 2.08 & 0.35 & 0.65 \\
24 quarters & 0.74 & 1.80 & 0.30 & 0.74 \\
\hline \multicolumn{5}{r}{ Adaptive expectations model } \\
\hline Forecast Horizon & Consumption & Investment & Hours & Real wage \\
1 quarter & 0.05 & 4.29 & 1.05 & 0.05 \\
4 quarters & 0.34 & 3.21 & 0.71 & 0.34 \\
8 quarters & 0.61 & 2.36 & 0.58 & 0.61 \\
16 quarters & 0.83 & 1.78 & 0.53 & 0.83 \\
24 quarters & 0.93 & 1.56 & 0.52 & 0.93 \\
\hline
\end{tabular}


TABLE 6. Cumulative responses relative to output following the biased technology shock: indivisible labor $(\eta=0)$

\begin{tabular}{lllll}
\hline \hline \multicolumn{5}{c}{ Rational expectations model } \\
\hline Forecast Horizon & Consumption & Investment & Hours & Real wage \\
1 quarter & 0.43 & 5.46 & 1.43 & 0.43 \\
4 quarters & 0.25 & 4.91 & 1.25 & 0.25 \\
8 quarters & 0.23 & 4.29 & 1.05 & 0.23 \\
16 quarters & 0.42 & 3.37 & 0.76 & 0.42 \\
24 quarters & 0.57 & 2.78 & 0.65 & 0.57 \\
\hline \multicolumn{5}{r}{ Adaptive expectations model } \\
\hline Forecast Horizon & Consumption & Investment & Hours & Real wage \\
1 quarter & 0.43 & 5.46 & 1.43 & 0.43 \\
4 quarters & 0.36 & 4.76 & 1.20 & 0.36 \\
8 quarters & 0.59 & 4.71 & 1.25 & 0.59 \\
16 quarters & 0.95 & 5.28 & 1.48 & 0.95 \\
24 quarters & 1.16 & 5.69 & 1.63 & 1.16 \\
\hline
\end{tabular}

TABLE 7. Relative contribution of biased technology shocks with indivisible labor $(\eta=0)$ : ratio of learning to $\mathrm{RE}$

\begin{tabular}{lllll}
\hline \hline Forecast Horizon & Output & Consumption & Investment & Hours \\
1 quarter & 1.69 & 2.26 & 1.10 & 1.00 \\
4 quarters & 1.52 & 2.96 & 1.10 & 1.00 \\
8 quarters & 1.44 & 3.32 & 1.12 & 1.00 \\
16 quarters & 1.38 & 1.78 & 1.15 & 1.00 \\
24 quarters & 1.35 & 1.32 & 1.16 & 1.00 \\
\hline
\end{tabular}


Responses to a Neutral Technology Shock: RE vs. Learning
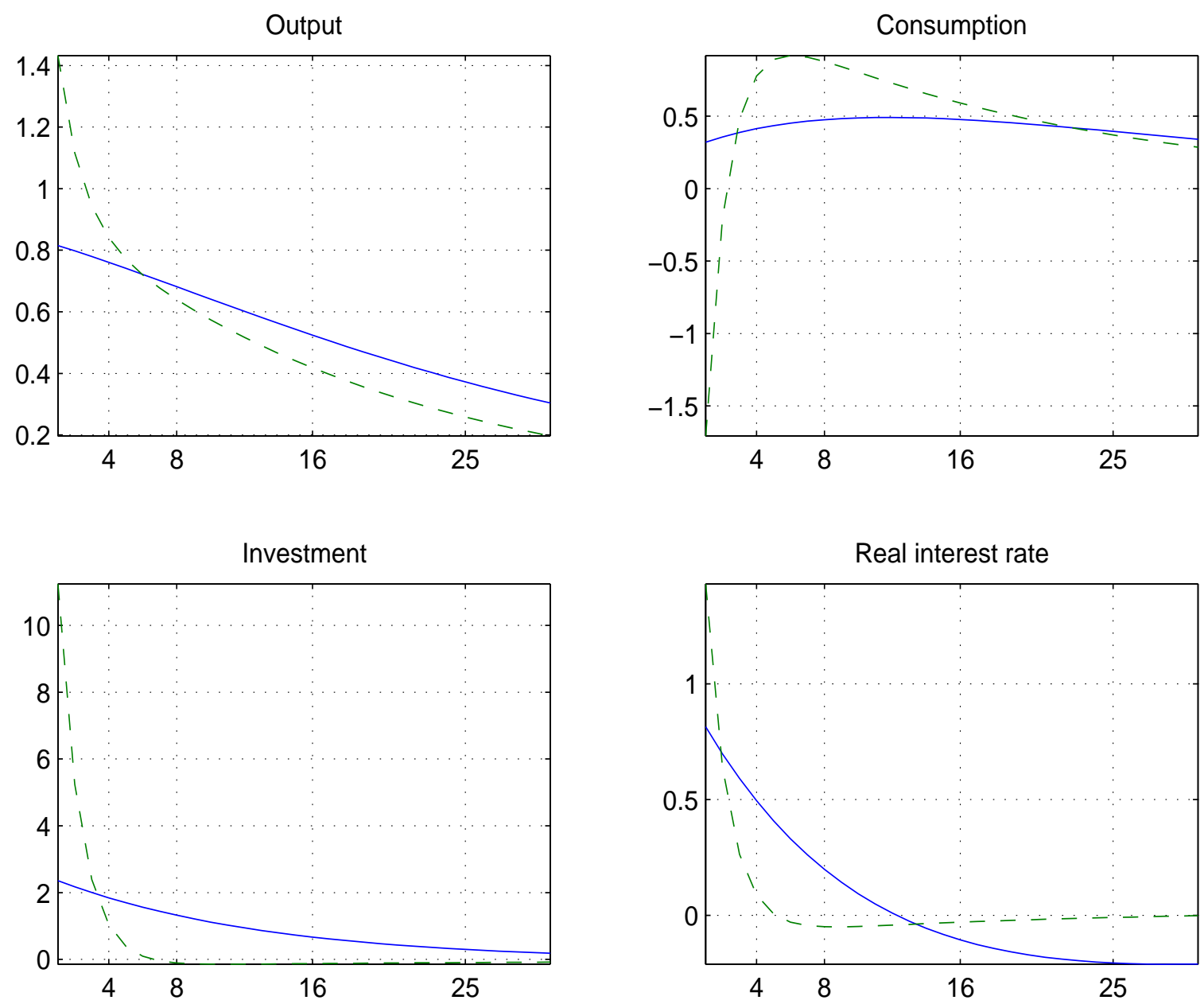

Hours

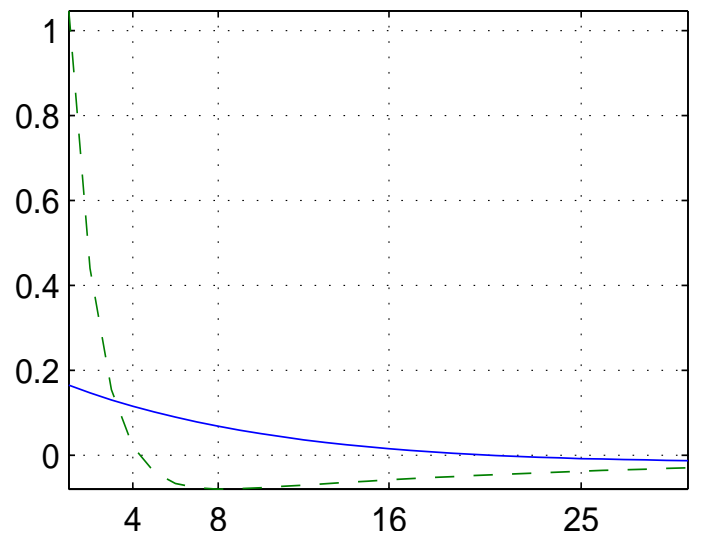

Real wage

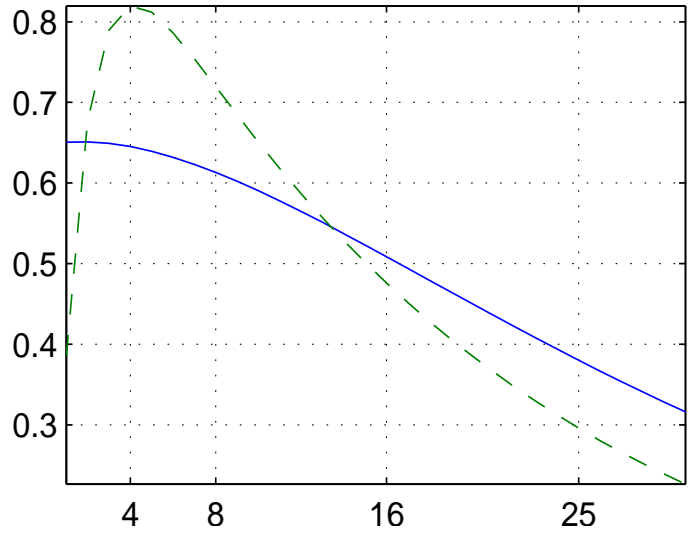

Figure 1. Impulse responses to a neutral technology shock in the benchmark model. The solid line represents the responses from the model with rational expectations. The dashed line represents the responses from the model with adaptive expectations. 
Responses to a Biased Technology Shock: RE vs. Learning
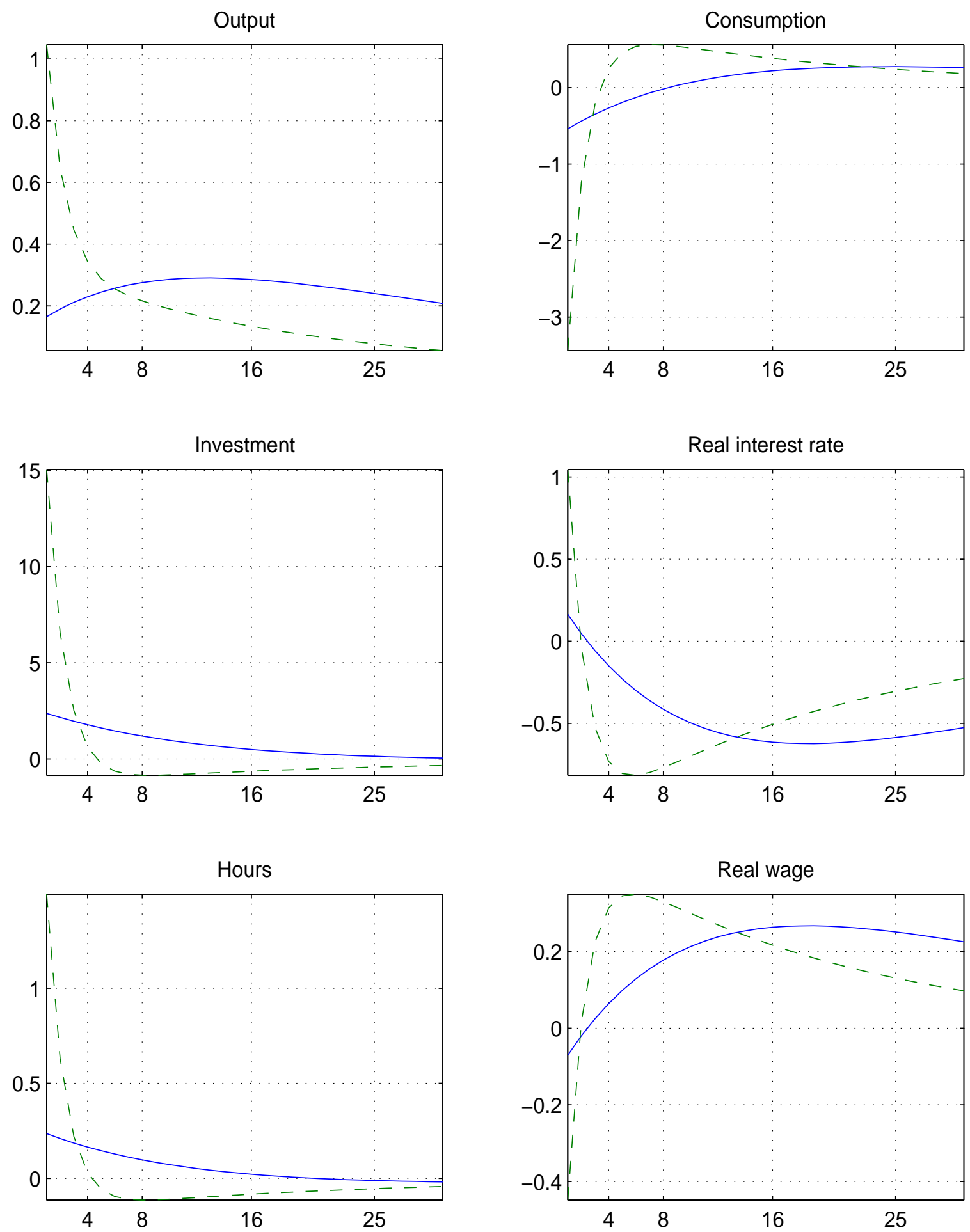

Figure 2. Impulse responses to a biased technology shock in the benchmark model. The solid line represents the responses from the model with rational expectations. The dashed line represents the responses from the model with adaptive expectations. 
Responses to a Neutral Technology Shock: RE vs. Learning

Output

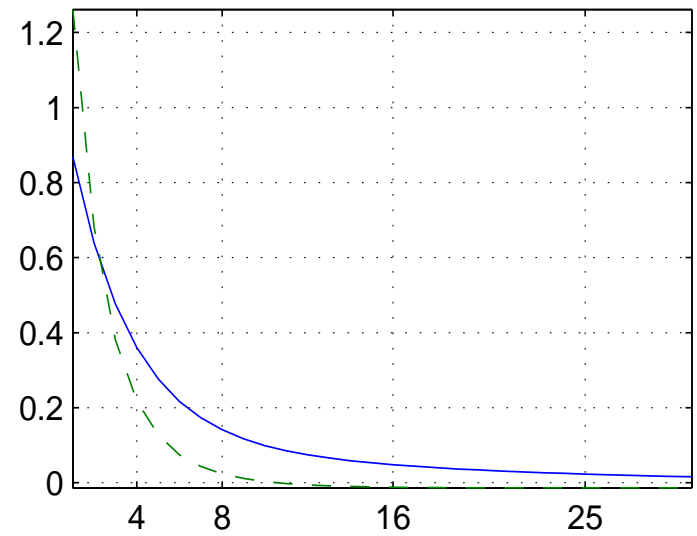

Investment

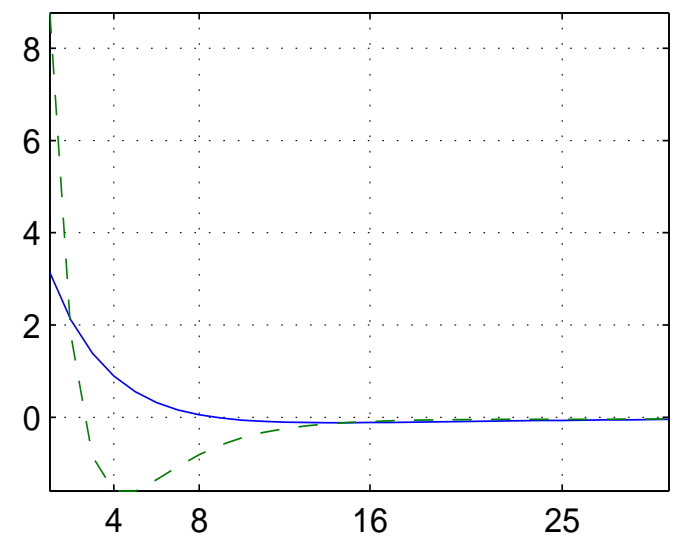

Hours

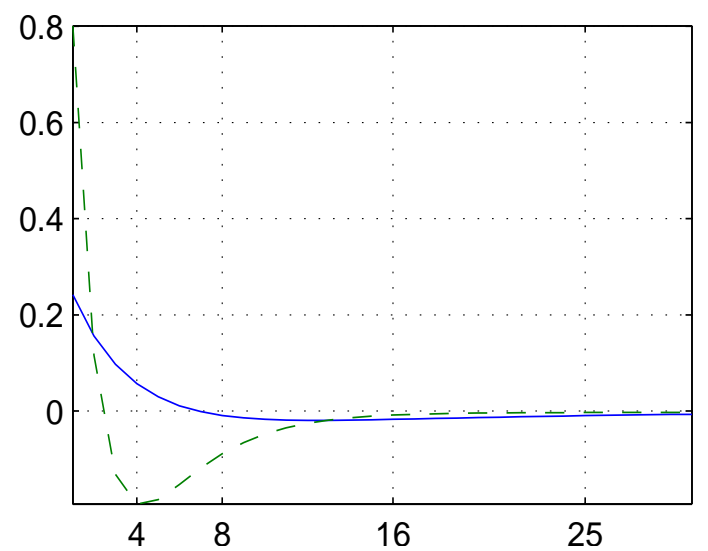

Consumption

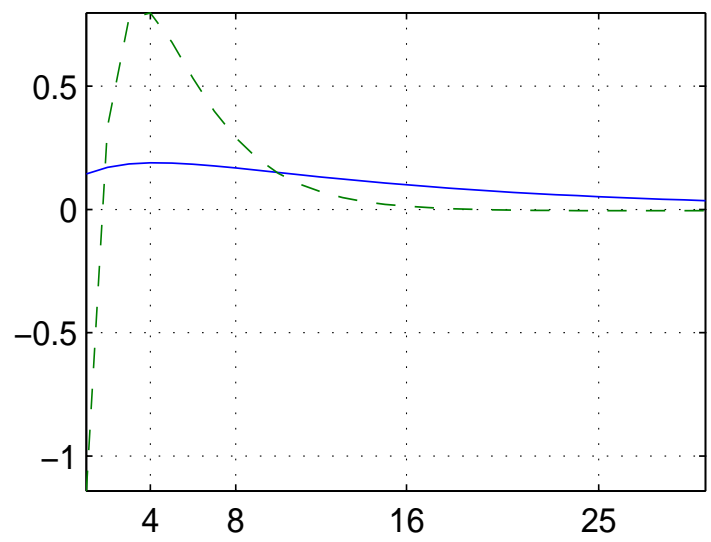

Real interest rate

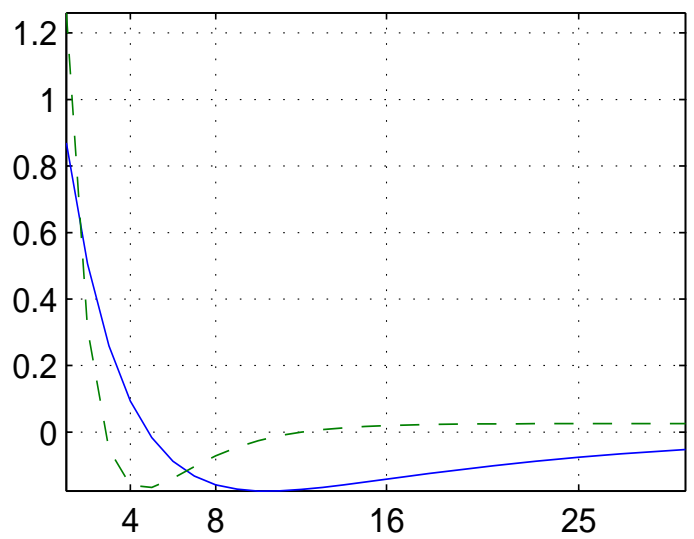

Real wage

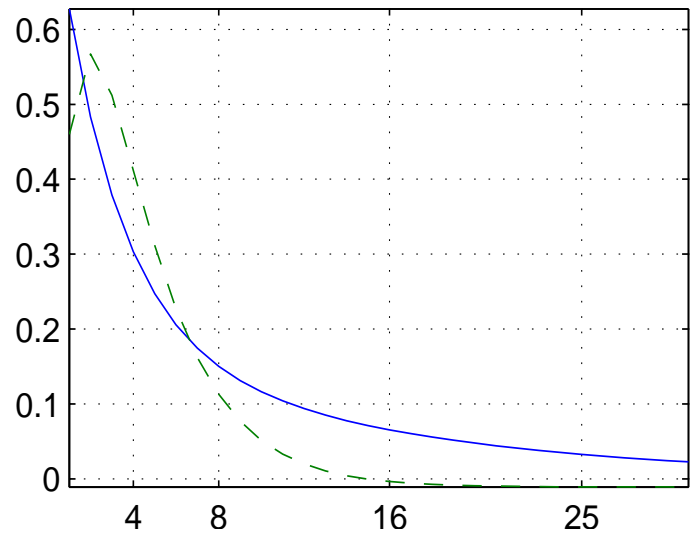

Figure 3. Impulse responses to a neutral technology shock with low persistence of the shock. The solid line represents the responses from the model with rational expectations. The dashed line represents the responses from the model with adaptive expectations. 
Responses to a Biased Technology Shock: RE vs. Learning

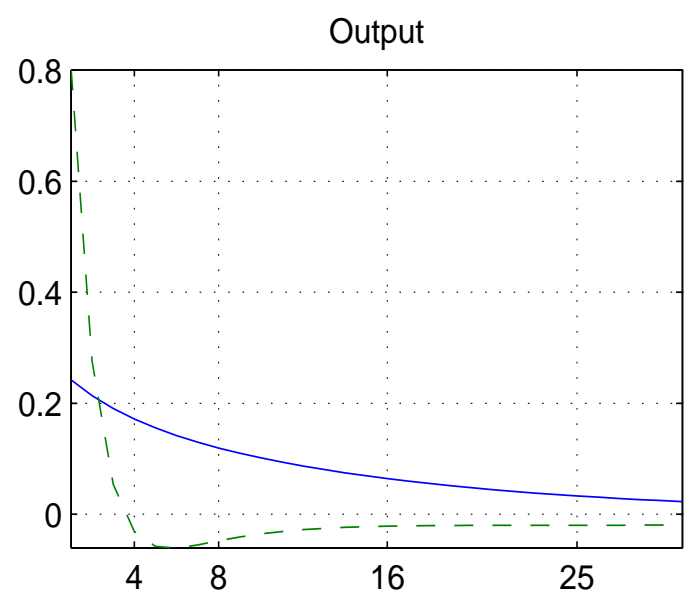

Consumption

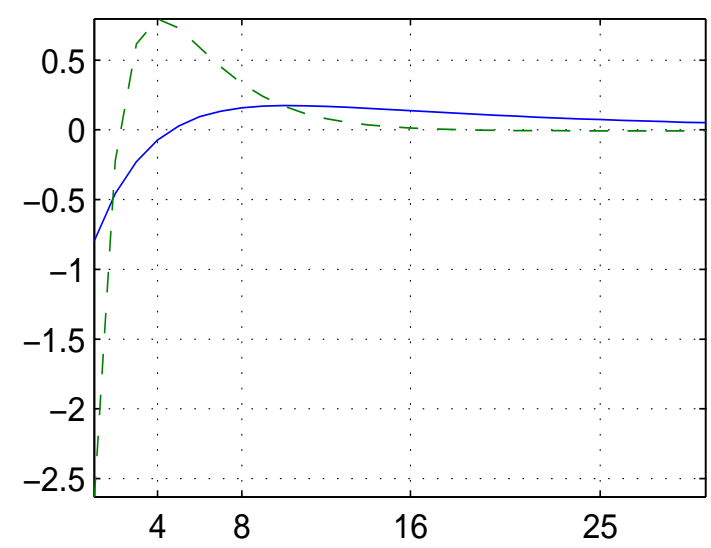

Investment

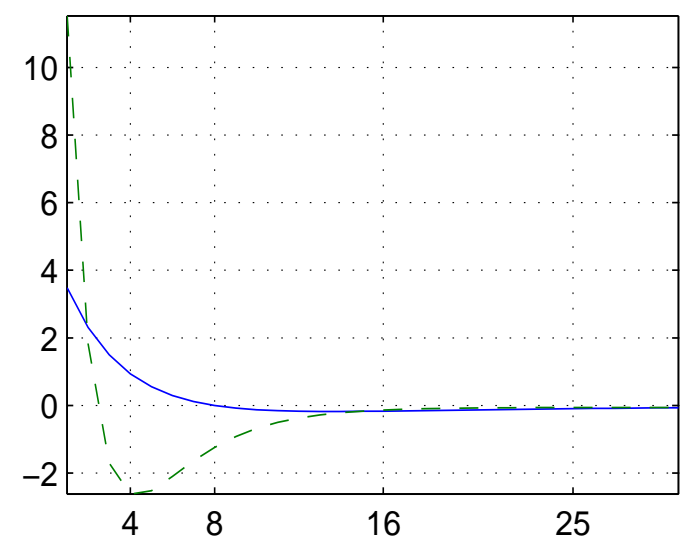

Real interest rate

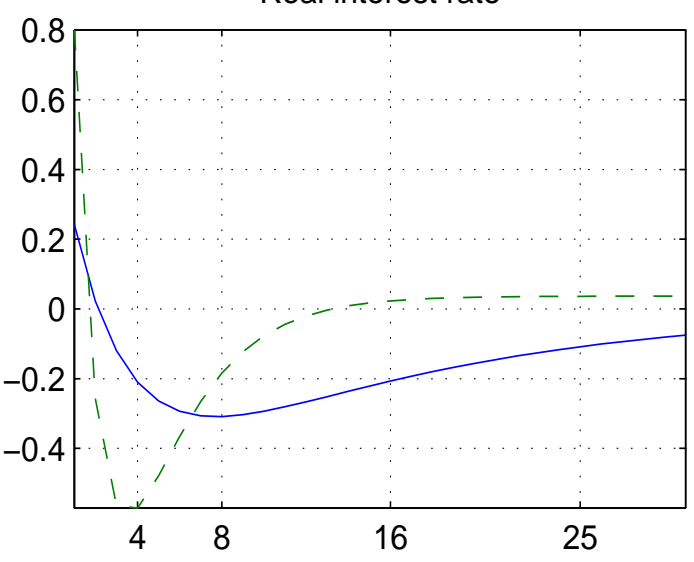

Hours

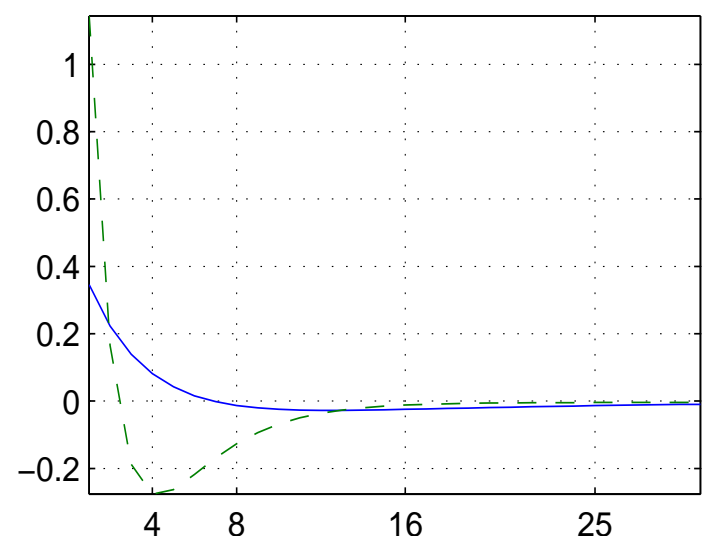

Real wage

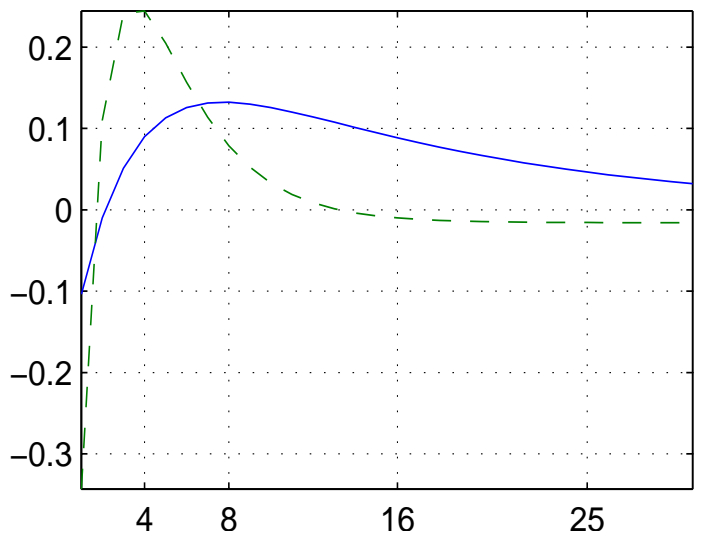

FIGURE 4. Impulse responses to a biased technology shock with low persistence of the shock. The solid line represents the responses from the model with rational expectations. The dashed line represents the responses from the model with adaptive expectations. 
Responses to a Neutral Technology Shock: RE vs. Learning

Output

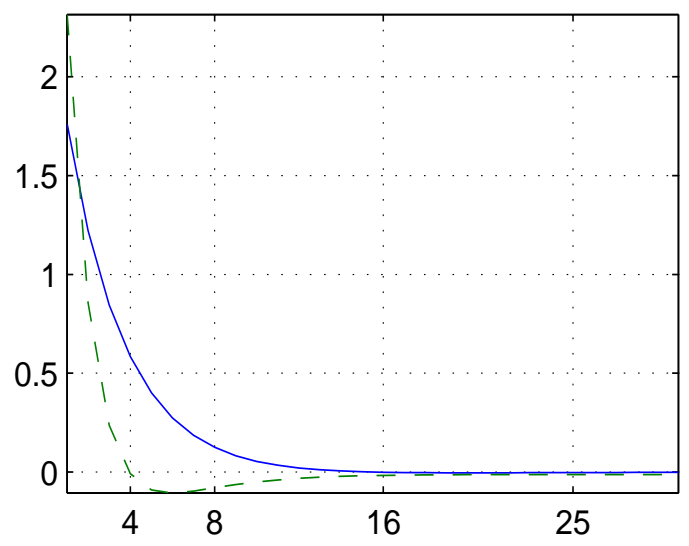

Investment

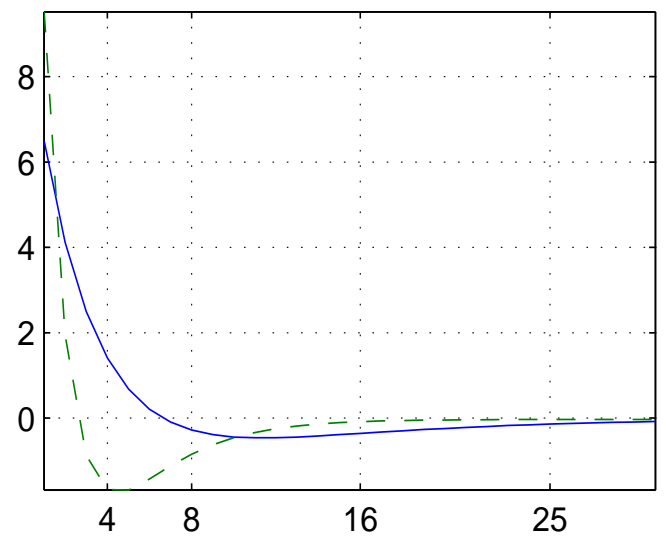

Hours

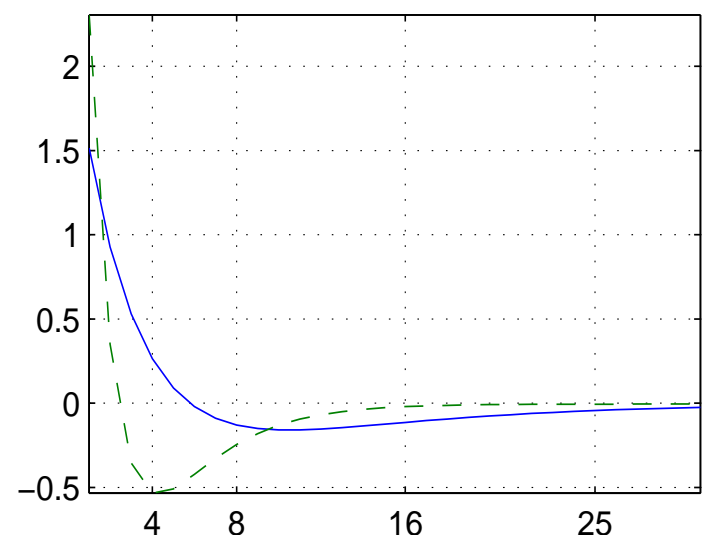

Consumption

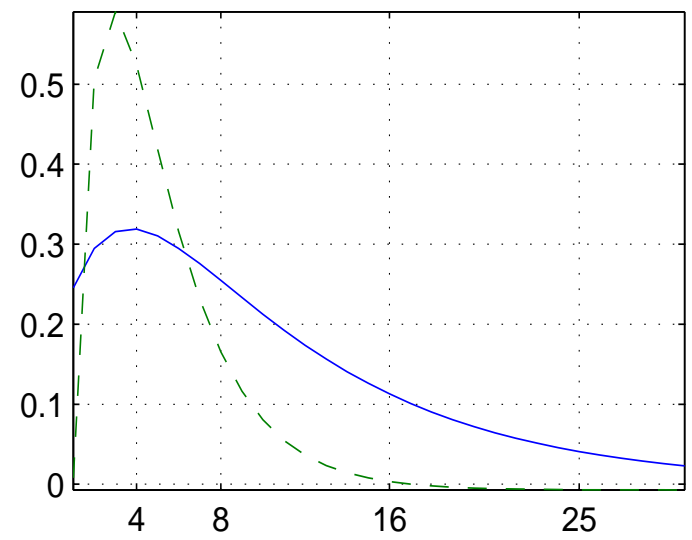

Real interest rate

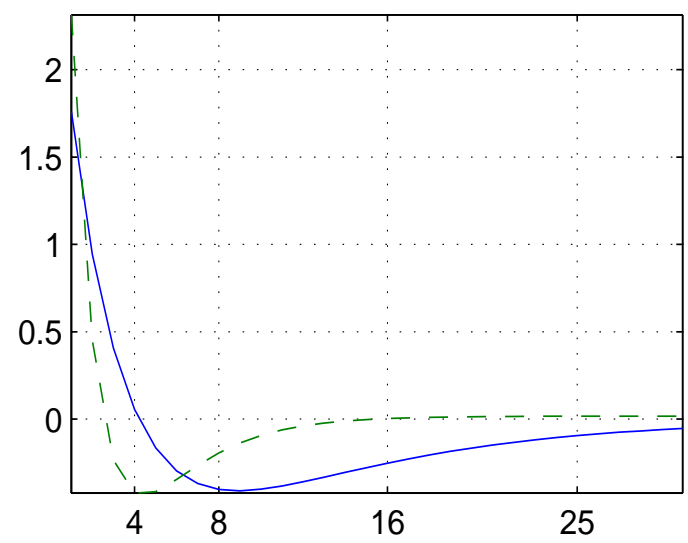

Real wage

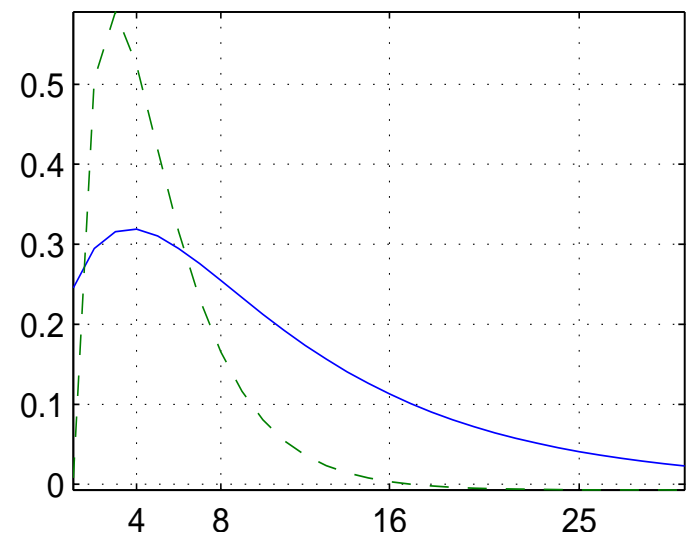

Figure 5. Impulse responses to a neutral technology shock with low persistence in the model with indivisible labor. The solid line represents the responses from the model with rational expectations. The dashed line represents the responses from the model with adaptive expectations. 


\section{Responses to a Biased Technology Shock: RE vs. Learning}
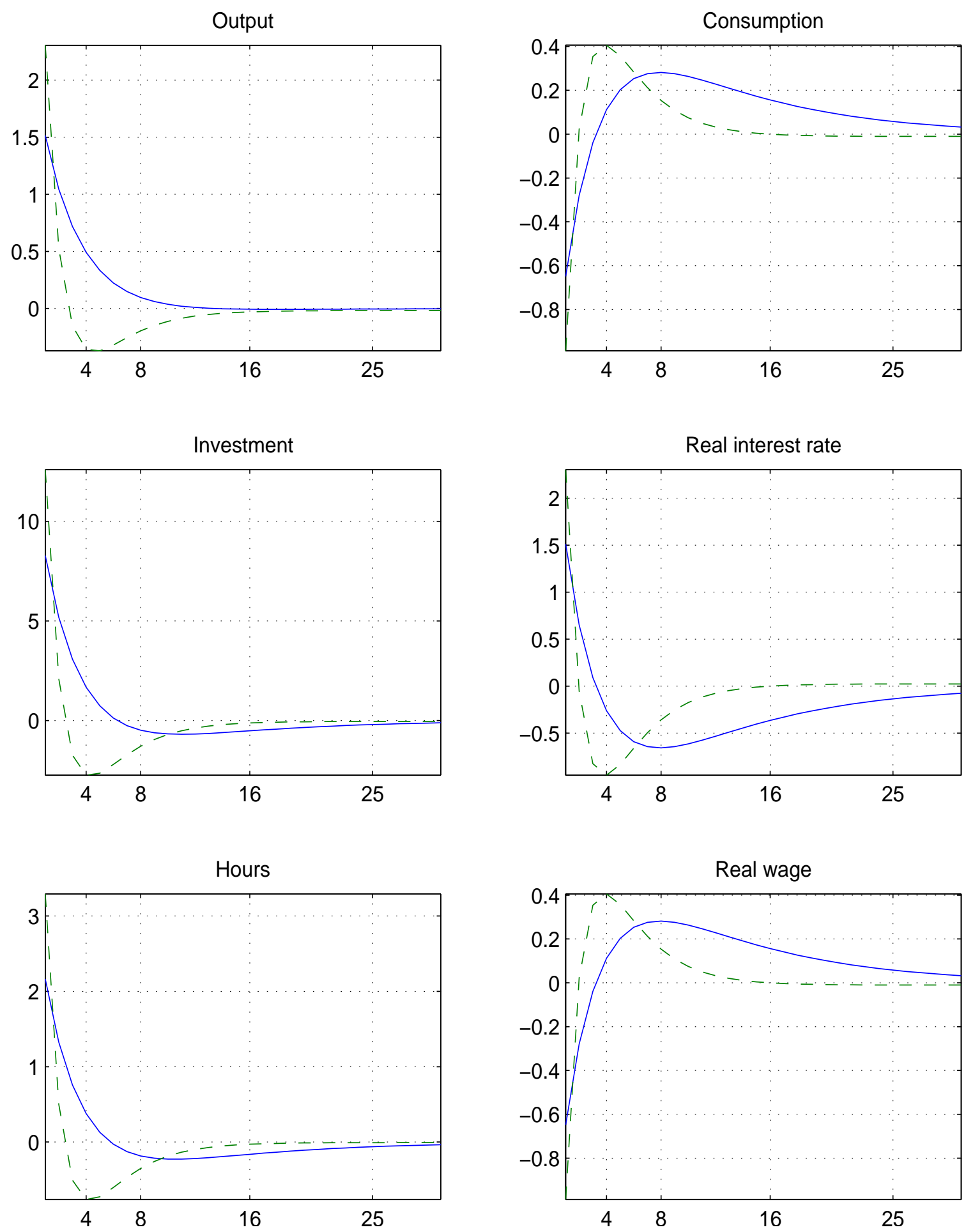

Figure 6. Impulse responses to a biased technology shock with low persistence in the model with indivisible labor. The solid line represents the responses from the model with rational expectations. The dashed line represents the responses from the model with adaptive expectations. 


\section{REFERENCES}

Adam, K., G. W. Evans, and S. Honkapohja (2006): "Are Hyperinflation Paths Learnable?," Journal of Economic Dynamics and Control, 30, 2725-2748.

Adam, K., A. Marcet, And J. P. Nicolini (2008): "Stock Market Volatility and Learning," Unpublished Manuscript, Universitat Pompeu Fabra.

Bullard, J., And J. Duffy (2004): "Learning and Structural Change in Macroeconomic Data," Working Paper 2004-016, Federal Reserve Bank of St Louis.

Carboni, G., and M. Ellison (2008): "The Great Inflation and the Greenbook," Manuscript, European Central Bank and University of Oxford.

Cho, I.-K., N. Williams, and T. J. Sargent (2002): "Escaping Nash Inflation," Review of Economic Studies, 69, 1-40.

Christiano, L., and M. Eichenbaum (1992): "Current Real Business Cycle Theories and Aggregate Labor Market Fluctuations," American Economic Review, 82(3), 430-450.

Evans, G., And S. HonkapohJA (2001): Learning and Expectations in Macroeconomics. Princeton University Press, Princeton, New Jersey.

Fisher, J. D. (2006): "The Dynamic Effects of Neutral and Investment-Specific Technology Shocks," Journal of Political Economy, 114, 413-452.

Greenwood, J., Z. Hercowitz, and P. Krusell (1997): "Long-Run Implications of Investment-Specific Technological Change," American Economic Review, 87, 342-362.

(2000): "The Role of Investment-Specific Technological Change in the Business Cycle," European Economic Review, 44, 91-115.

Hansen, G. D. (1985): "Indivisible Labor and the Business Cycle," Journal of Monetary Economics, 16, 309-337.

He, H., And Z. Liu (2008): "Investment-Specific Technological Change, Skill Accumulation, and Wage Inequality," Review of Economic Dynamics, 11, 314-334.

KASA, K. (2004): "Learning, Large Deviations, And Recurrent Currency Crises," International Economic Review, 45, 141-173.

Krusell, P., L. E. Ohanian, J. V. Ríos-Rull, and G. L. Violante (2000): "Capital-Skill Complementarity and Inequality: A Macroeconomic Analysis," Econometrica, 68(5), 1029-1053. 
Kushner, H. J., And G. G. Yin (1997): Stochastic Approximation Algorithms and Applications. Springer-Verlag.

LuCAS, JR., R. E. (1986): "Adaptive Behavior and Economic Theory," Journal of Business, 59, S401-S426.

Marcet, A., And J. P. Nicolini (2003): "Recurrent Hyperinflations and Learning," American Economic Review, 93(5), 1476-1498.

Marcet, A., and T. J. Sargent (1989): "Convergence of Least Squares Learning Mechanisms in Self-Referential Linear Stochastic Models," Journal of Economic Theory, 48, 337-368.

Pencavel, J. H. (1986): "The Labor Supply of Men: A Survey," in Handbook of Labor Economics, ed. by O. C. Ashenfelter, and R. Layard, pp. 3-102. Elsevier, Amsterdam, North Holland.

Primiceri, G. (2006): "Why Inflation Rose and Fell: Policy-Makers' Beliefs and U. S. Postwar Stabilization Policy," Quarterly Journal of Economics, 121(3), 867-901. Rogerson, R. (1988): "Indivisible Labor, Lotteries and Equilibrium," Journal of Monetary Economics, 21, 3-16.

Sargent, T. J. (1999): The Conquest of American Inflation. Princeton University Press, Princeton, New Jersey.

(2007): "Evolution and Intelligent Design," AEA Presidential Address, New York University.

Sargent, T. J., N. Williams, and T. Zha (2006a): "The Conquest of South American Inflation," NBER Working Paper No. 12606.

(2006b): "Shocks and Government Beliefs: The Rise and Fall of American Inflation," American Economic Review, 96(4), 1193-1224.

Williams, N. (2003): "Adaptive Learning and Business Cycles," Manuscript, Princeton University.

Vanderbilt University, Emory University, Federal Reserve Bank of Atlanta 